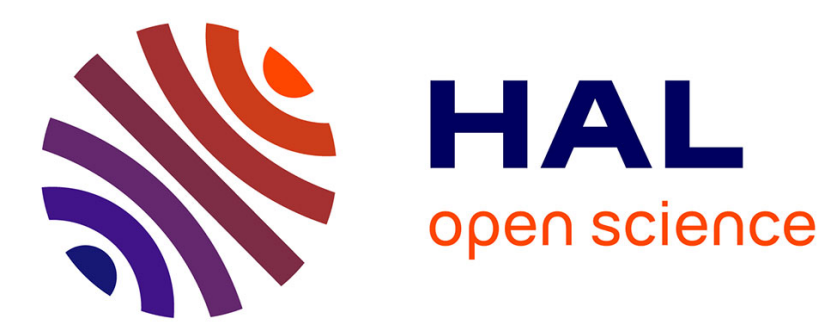

\title{
The impact of circularity defects on bridge stay cable dry galloping stability
}

A. Benidir, O. Flamand, G Dimitriadis

\section{To cite this version:}

A. Benidir, O. Flamand, G Dimitriadis. The impact of circularity defects on bridge stay cable dry galloping stability. Journal of Wind Engineering and Industrial Aerodynamics, 2018, 181 (181), p. 14-26. 10.1016/j.jweia.2018.08.009 . hal-02370599

\section{HAL Id: hal-02370599 \\ https://hal-cstb.archives-ouvertes.fr/hal-02370599}

Submitted on 19 Nov 2019

HAL is a multi-disciplinary open access archive for the deposit and dissemination of scientific research documents, whether they are published or not. The documents may come from teaching and research institutions in France or abroad, or from public or private research centers.
L'archive ouverte pluridisciplinaire HAL, est destinée au dépôt et à la diffusion de documents scientifiques de niveau recherche, publiés ou non, émanant des établissements d'enseignement et de recherche français ou étrangers, des laboratoires publics ou privés. 
The impact of circularity defects on bridge stay cable dry galloping stability.

\author{
A. Benidir ${ }^{1}$, O. Flamand $^{2}$, G. Dimitriadis ${ }^{3}$ \\ 1. National Centre of Integrated Studies and Research on Building Engineering (CNERIB), \\ New El Mokrani city, 16201, Souidania, Algiers, Algeria \\ abenidir.cnerib@gmail.com
}

\author{
2. CSTB, 11 rue Henri PICHERIT 44300 Nantes France \\ 3. Aerospace and Mechanical Engineering Department, University of Liège, 4000 \\ Liège, Belgium
}

Keywords: Circular cylinder; shape distortion; dry galloping; bi-stability; critical regime.

\begin{abstract}
The present work studies the influence of circularity defects, on the aerodynamic behaviour of stay cables of cable-stayed bridges. It focuses on wind tunnel tests on High-Density Polyethylene cable covers with and without helical fillets, in a range of Reynolds numbers from the sub-critical to the critical regime. The paper considers the impact of circularity defects on the aerodynamic stability of cable sheaths by testing various amplitudes of imposed ovalization. The defects are artificially applied on real sheaths whose original cross-sections are close to circular. The experiment consists in measuring surface pressures to investigate how the amplitude of ovalization influences the flow around the sheaths, especially in the critical Reynolds number regime when transition in the boundary layer occurs. The analysis is based on bifurcation diagrams and Proper Orthogonal Decomposition. The investigation demonstrates that important circularity defects can significantly increase the bistable nature of the flow around a sheath at the critical regime. Nevertheless, the introduction of a helical fillet de-correlates the flow around the sheath, causing jumps in lift that have different sign along its length.
\end{abstract}

\title{
1. INTRODUCTION
}

The underlying mechanism that causes dry galloping of bridge stay cables is still under investigation by several research teams. Dry galloping causes significant vibrations of inclined cables under dry conditions, exclusively due to wind (Nikitas et al. (2015)). Field observation, numerical simulation and wind tunnel tests have been used to identify the parameters that affect these vibrations. Previous studies reported that the origin of dry galloping of stay cables is related to the flow around the cable sheath at the critical flow regime (Mctavish et al. (2017), Larose and Zan (2001), Cheng et al. (2003)). These high amplitude vibrations are serious concerns for bridge safety. Most cable sheaths used in the US and Europe are either smooth or feature helical fillets, while tubes with pattern-indented surfaces are mainly used in Asia (Kleissl and Georgakis (2012)). Katsuchi et al. (2009) investigated the mechanism of dry galloping in both smooth and pattern-indented surfaces. The authors reported large cable vibrations in models with these surfaces at different Reynolds numbers. The findings exhibited the importance of the cross-section and/or the surface parameters on triggering cable vibrations near the critical regime. Furthermore, dry galloping has been proved to occur in a critical range of yaw/inclination angle and at a specific reduced wind speed (Vo et al. (2016)). In wind tunnel tests, it is 
possible to reproduce large cable vibrations at particular orientations. In general, the damping of the models used in such tests is low. In field observation, cable vibration amplitudes up to $1.5 \mathrm{~m}$ have been observed in Japan in windy and dry weather conditions (Matsumoto et al. (2010)). The estimated wind velocity was close to $18 \mathrm{~m} / \mathrm{s}$, which coincides with the beginning of the critical flow regime around these specific cables. In this flow regime, Karman vortex mitigation occurs and the drag crisis phenomenon is observed. The latter is related to the transition from laminar to turbulent flow in the boundary layers (Transition in Boundary Layer - Zdravkovich (1997)). The rapid drop in the drag coefficient is combined with the appearance of a non-negligible lift force, which is a consequence of the appearance of a negative pressure bubble on one side of the circular cylinder. Schewe (1983) used the term bi-stability to describe the occurrence of transition on either one side of a circular cylinder. Recent research conducted by Nikitas et al. (2012) and Benidir et al. (2014) showed that bi-stability causes abrupt jumps between the different regimes of the boundary layers at constant Reynolds number. However, as the dry galloping mechanism is referenced it has an aerodynamic origin; this consideration just sheds light on an important parameter, which is the macroscopic defect of the cable sheaths. The impact of the real circularity defect on flow around circular cylinders was investigated first by Flamand and Boujard (2009), then by Matteoni and Georgakis (2012). Benidir et al. (2015) measured the circularity defects on four smooth High-Density Polyethylene sheaths of different diameter. They carried out wind tunnel tests to show that this defect influences the flow around the sheaths, especially at the critical regime. Ma et al. (2015) asserted that the aerodynamic forces on cables are considerably affected by the angle of attack, especially in the critical regime. Their tests were carried out on smooth cable surfaces with elliptical and semi-elliptical cross-sections.

The present work aims to investigate the impact of increasing the circularity defect of the cable sheath on the aerodynamic behaviour of stay cables by means of bifurcation diagrams and Proper Orthogonal Decomposition (POD). The paper also presents the comparison between the pressure patterns of smooth and helical fillet cable sheaths at the critical flow regime. Finally, the work investigates the impact of large deformations of the sheaths on the effect of the helical fillet.

\section{EXPERIMENTS}

\subsection{Setup}

The $4 \mathrm{~m} \times 2 \mathrm{~m}$ wind tunnel of the Scientific and Technical Centre for Building (CSTB) was used for full-scale measurements of surface pressures on cable models. Wind speed was varied from 0 to 28 $\mathrm{m} / \mathrm{s}$, corresponding to a Reynolds number range between $1.03 \times 10^{5}$ and $4.04 \times 10^{5}$. Static tests were performed on High-Density Polyethylene (HDPE) smooth and helical fillet cable covers normal or inclined to the flow with a mean diameter $\mathrm{D}=250 \mathrm{~mm}$. The results of the experiment on the smooth cables are reported in Benidir et al. (2015). A 3D surface characterization device was used to measure the surface roughness. The average roughness values ( $\mathrm{Ra}$ ) of the covers are $1.29 \mu \mathrm{m}$ and $1.43 \mu \mathrm{m}$, respectively for smooth sheaths and for sheaths with helical fillet surfaces. The turbulence intensity of the wind tunnel was measured by a Cobra probe and was found to be less than $1.1 \%$ at the location of the model. The model length was $\mathrm{L}=2 \mathrm{~m}$, representing an aspect ratio of $\mathrm{L} / \mathrm{D}=8 \%$ and a wind tunnel blockage factor of $\mathrm{D} / \mathrm{W}=6.2 \%$, where $\mathrm{W}=4 \mathrm{~m}$ is the width of the wind tunnel. In addition, the instrumented part of the models was located far from the walls, in the middle of the working section. The helical fillets were $1 \mathrm{~mm}$ high and $3 \mathrm{~mm}$ wide and were inclined by $45^{\circ}$ to the longitudinal axis of the cylinders. The lengthwise spacing of the helical fillet was $550 \mathrm{~mm}$. Figure 1 -a shows the helical fillet HDPE cover in the wind tunnel's test section. 
Each sheath was equipped with 288 pressure taps distributed around 9 rings (figures $\underline{1}$-a and figure $\underline{1}$ b) in the central part of the tube. The distance between the bottom ring (ring number 1) and the top ring (ring number 9) was $1 \mathrm{~m}$. The distribution of the rings was not equidistant along the sheath; the largest separation between two successive rings was $20 \mathrm{~cm}$ while the smallest was $5 \mathrm{~cm}$. Thus, the concentration of the pressure taps was higher close to the mid-section of the tube. Each pressure tap was connected via a short tube to a pressure sensor with a measurement range of $2500 \mathrm{~Pa}$. These sensors were collected in groups of 32 in a pressure scanner.

A laser displacement sensor with a resolution of $60 \mu \mathrm{m}$ was used to measure the original circularity defects of the tubes. Each tube was rotated at a steady rate around its axis and the laser sensor was placed normal to the surface at a suitable distance.

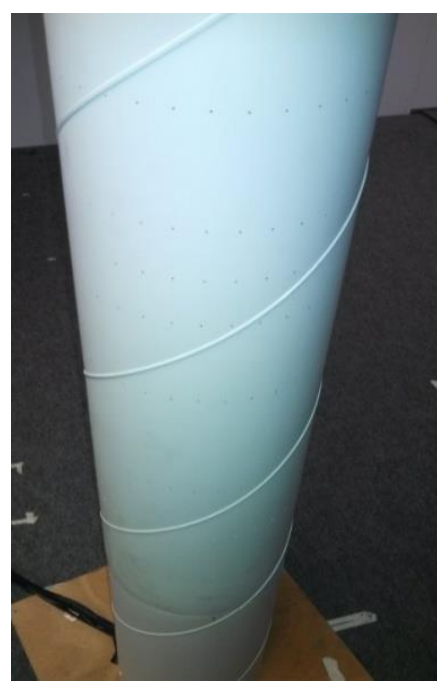

(a)

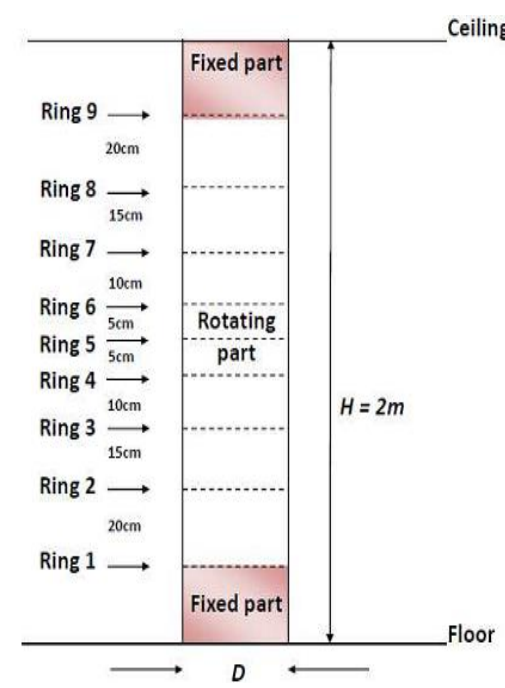

(b)

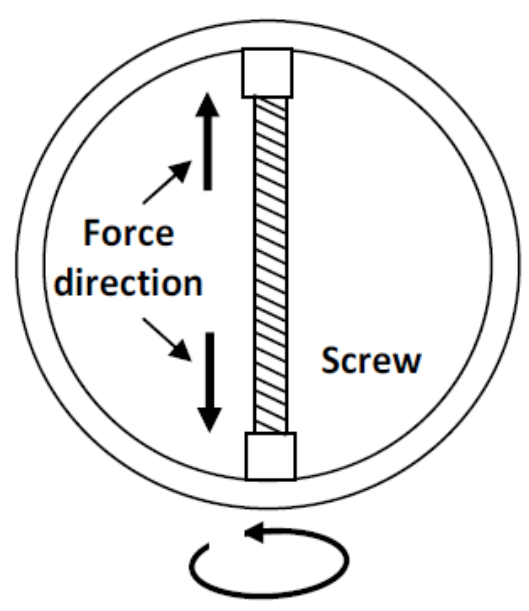

(c)

Figure 1: (a) HDPE cover with helical fillets, diameter D = 250mm; (b) 288 pressure taps (9 rings of 32 pressure taps); (c) mechanism designed for tuning the ovalization of HDPE tube.

A second set of tests was carried out in order to determine the effect of the tubes' cross-sectional shape. A controlled deformation was applied on the smooth and helical fillet cable sheaths with the same diameter. The cross-sectional shape distortion procedure consisted of an application of a decompression force inside the cylinder to strain the section in one direction. The mechanism was composed of two metallic reinforcement bars placed inside each cylinder along its axis (figure $1-\mathrm{c}$ ). A distortion force was applied by means of five threaded shafts equally spaced along the length of the reinforcement bars. The resulting circularity defect of the two cylinders was controlled with a caliper. The mechanism was used for generating ovalization defects up to $7 \mathrm{~mm}$ with respect to the original diameter.

\subsection{Measurement protocol}

The objective of the experiments was to study the impact of surface distortion for different wind directions. Thus, for the smooth and helical fillet cable sheaths respectively,16 and 9 wind incidences (i.e. angles of rotation around the longitudinal axis) were tested in the wind tunnel. The step change of the rotation angle was $22.5^{\circ}$ during all the tests. Flamand and Boujard (2009), followed by Matteoni and Georgakis (2012) and finally Benidir et al. (2014) confirmed the importance of wind incidence in the critical regime when the model is a HDPE sheath with natural defects. The angle of incidence is 
denoted by $\theta$. For instance, when $\theta=0^{\circ}$ or $\theta=22.5^{\circ}$, it indicates that the wind blows towards taps 1 and 3 respectively. By referring to the DIN8074 standard, the natural circularity defect of cable protection used for cable-stayed bridges is assumed to be less than $1 \%$ of the diameter. In this experiment, the natural defect is artificially increased up to $4.4 \%$ of the diameter.

\section{CIRCULARITY DEFECT MEASUREMENT}

The results of the circularity defect measurements for both kinds of cable surfaces are plotted in figure $\underline{2}$ and figure $\underline{3}$. Figure 2-a shows the cross-sectional shape of the smooth sheath at ring number 5 for four different distortion forces. Figure 2-b plots the cross-sectional shape at all the rings for the highest distortion force. Similarly, figures $\underline{3}-\mathrm{a}$ to $\underline{3}-\mathrm{d}$ plot increasing imposed circularity defects for the sheath with helical fillet. For both types of sheath the deformation ranges from natural (labelled 'Natural defect' in figure 2-a) to $4.4 \%$ of the diameter (labelled Dmax=257). The distortion criterion is taken to be the amplitude of deformation along the major axis of the oval shape, called the axis of deformation. Three amplitudes of deformation have been achieved, equal to $253 \mathrm{~mm}, 255 \mathrm{~mm}$ and $257 \mathrm{~mm}$, as presented in figure 2-a and figure $\underline{3}$. As the length of the major axis increases, that of the minor axis decreases. For instance, when the major axis reaches Dmax $=257 \mathrm{~mm}$ the length of the minor axis is close to $245 \mathrm{~mm}$.

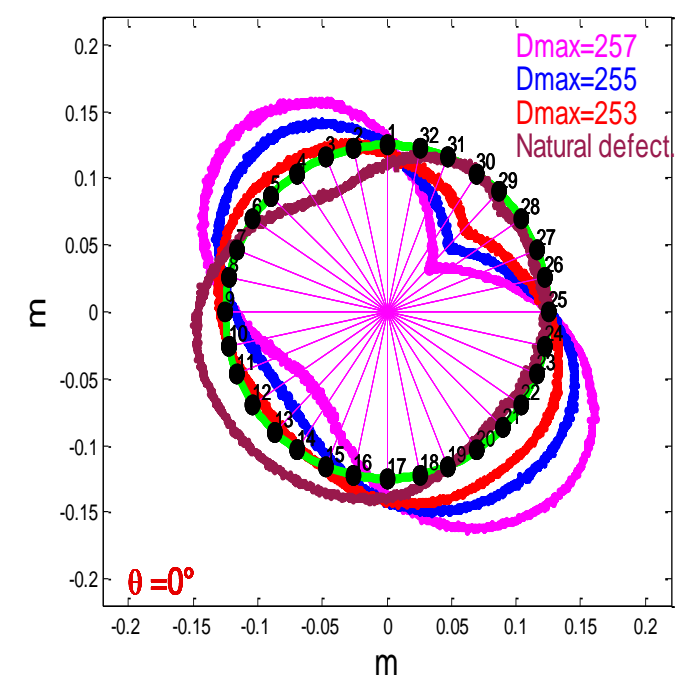

(a)

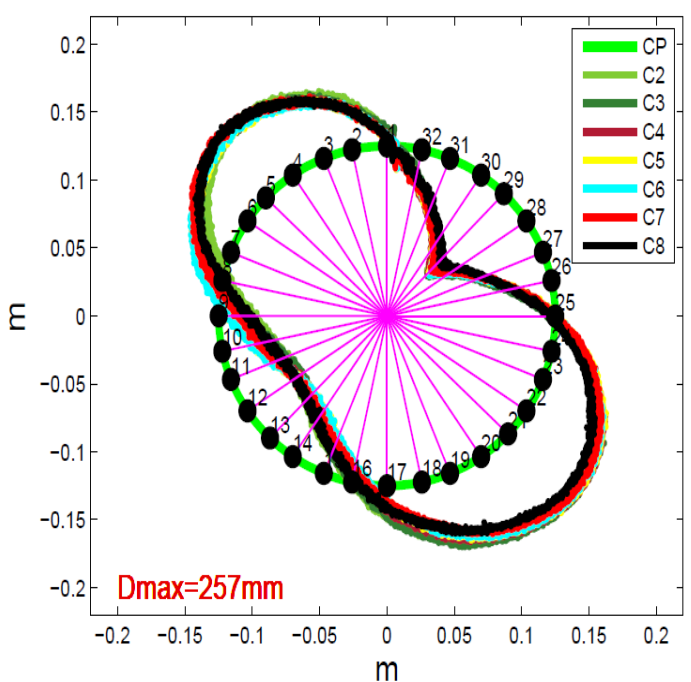

(b)

Figure 2: (a) Deformations of smooth cable surfaces, (b) Circularity defect variation along the length of the cable for Dmax $=257 \mathrm{~mm}$. 


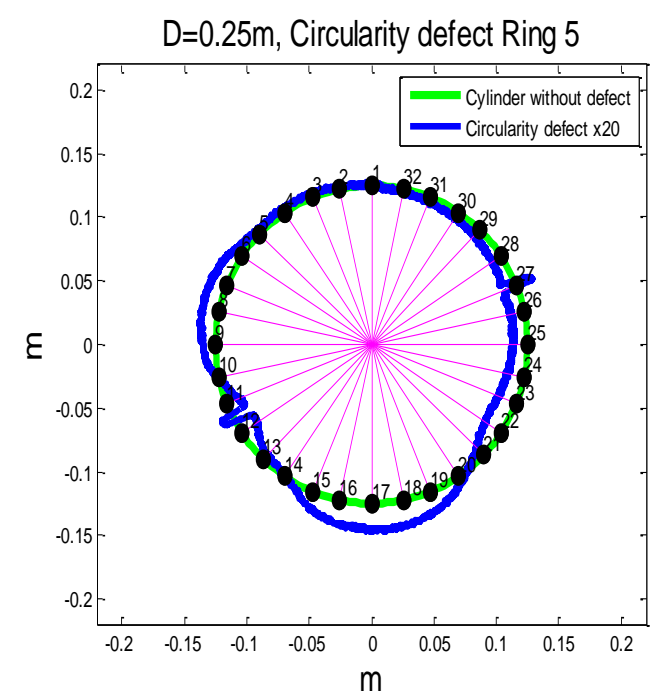

(a) Natural defect

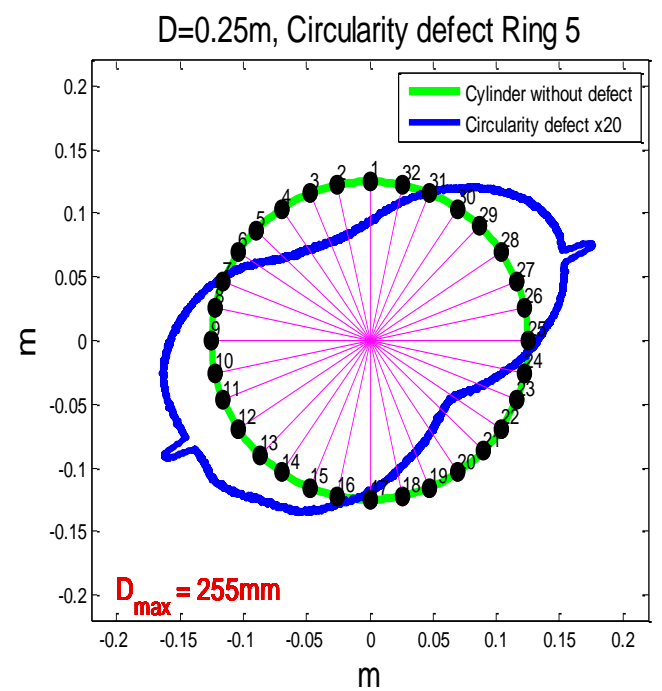

(c) $\operatorname{Dmax}=255 \mathrm{~mm}$

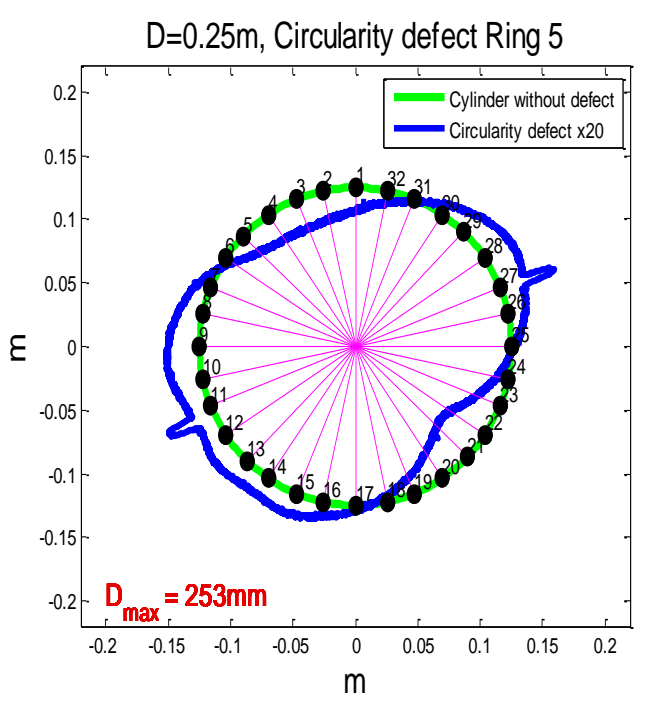

(b) $\operatorname{Dmax}=253 \mathrm{~mm}$

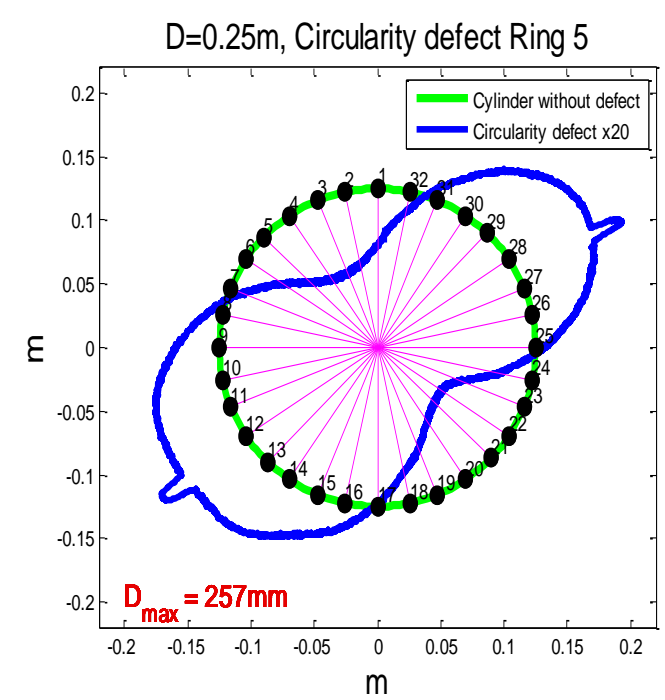

(d)Dmax $=257 \mathrm{~mm}$

Figure3: Natural and imposed circularity defect on sheath with helical fillet.

\section{RESULTS}

The impact of the shape distortion of smooth and non-smooth surfaces on the aerodynamic behaviour of cable sheaths is discussed below. The interpretation of the data is detailed in two parts. The first part is dedicated to the variation of the mean aerodynamic load coefficients and the second part analyses the instantaneous drag and lift forces inferred from the pressure tap time records.

\subsection{Mean force coefficient}

Mean aerodynamic load coefficients are obtained by integrating the instantaneous pressure distribution at each ring. Lift and drag coefficients are defined by

$$
C_{D}=\frac{F_{D}}{\frac{1}{2} \rho U^{2} S}
$$




$$
C_{L}=\frac{F_{L}}{\frac{1}{2} \rho U^{2} S}
$$

where $F_{D}$ and $F_{L}$ represent the drag and lift forces respectively, $\rho$ the air density, $U$ the airspeed and $S$ the reference surface. The reference surface $(\mathrm{S})$ is obtained by multiplying the original diameter $\mathrm{D}$ $(250 \mathrm{~mm})$ by the reference length $(\mathrm{L}=1 \mathrm{~m})$. $\mathrm{L}$ corresponds also to the length of the instrumented area. The forces $F_{D}$ and $F_{L}$ are obtained from

$$
\begin{aligned}
& F_{D}=\sum_{i=1}^{32} P_{i} x_{i} \\
& F_{L}=\sum_{i=1}^{32} P_{i} y_{i}
\end{aligned}
$$

where $x_{\mathrm{i}}$ and $y_{\mathrm{i}}$ are the characteristic areas of the $i$ th pressure tap projected in $\vec{x}$ (drag direction) and $\vec{y}$ (lift direction) respectively and $P_{\mathrm{i}}$ is the instantaneous pressure recorded at the $i$ th tap.

\subsubsection{Sheath with helical fillet}

Helical fillets are installed on bridge cable sheaths in order to avoid rain-and-wind-induced vibration Flamand (1995). The fillet tries to force the water rivulet to follow a modified direction and aims to de-correlate the fluctuations of the flow along the length of the sheath. The impact of natural circularity defect on cable sheath aerodynamics can be studied by comparing two different wind incidences at an angular distance of $180^{\circ}$ to each other. Figure $\underline{4}$ plots the mean lift coefficient as a function of Reynolds number at ring number 5 . The two wind incidences are $\theta=22.5^{\circ}$ and $\theta=202.5^{\circ}$, meaning that the wind blows directly towards taps 2 and 19 respectively. The cross-sectional shape seen by the oncoming flow is also depicted in each figure. The location of the helical fillet is identical for these two wind incidences, but the cable cross-sections are different due to the natural circularity defect. The mean lift coefficient calculated for these two cases shows a variation in the sign and the intensity of the lift force. In particular, the start of boundary layer transition occurs on opposite sides and the absolute value of the lift force measured for $\theta=22.5^{\circ}$ is $48 \%$ greater than that obtained for $\theta$ $=202.5^{\circ}$. It is worthwhile to note that the TrBL1 regime is established at different Reynolds numbers. These results show that the original circularity defect influences considerably the pressure pattern around the covers at the critical regime, even in the presence of the helical fillet.

The impact of large shape modification on the mean aerodynamic coefficients acting on the sheath is shown in figure $\underline{5}$. The variation of the mean drag and lift coefficients with Reynolds number at ring number 3 are given in figure $\underline{5}$-a and $\underline{5}$-b respectively. The wind is blowing directly towards pressure $\operatorname{tap} 3\left(\theta=22.5^{\circ}\right)$ and the cover shape is gradually distorted. The results around the original shape are also plotted. The original shape means that the ovalization setup is not yet installed inside the cable stay. It can be observed that the redistribution of the macroscopic defect around the cable induces a slight increase of the mean drag force at the sub-critical regime for this orientation. The graph shows also that the occurrence of the drag crisis, which indicates the presence of the TrBL1 regime, is shifted to higher Reynolds numbers as the distortion is increased. Additionally, the drop in drag is steeper for the undistorted section than for the distorted ones. In the super-critical regime, the drag coefficient again increases with distortion. For example, at a Reynolds number of $3.36 \times 10^{5}$ the drag coefficient 
is $C_{D}=0.44$ for the undistorted sheath; for the same Reynolds number but for Dmax $=257 \mathrm{~mm}$, the drag coefficient jumps to $C_{D}=0.52$, which constitutes an increase of $25 \%$.

In the critical regime, the drag crisis is accompanied by a sudden jump in the mean lift coefficient as shown in figure 5 -b. For the original cross-section, this jump appears at $\operatorname{Re}=2.25 \times 10^{5}$, which corresponds to the first drop of the drag. The beginning of the second transition is associated with the second drop in the drag force, noted as the transition from theTrBL1 to the TrBL2 regime. When this transition takes place, the lift forces decrease gradually, as can be observed for a Reynolds number higher than $2.4 \times 10^{5}$.

The increase and the reorganization of the circularity defect around the cover leads to a wider Reynolds number range in which boundary layer transition occurs. For the original section, the critical regime ranges from $\mathrm{Re}=2.25 \times 10^{5}$ to $\mathrm{Re}=2.88 \times 10^{5}$ while, for Dmax $=257 \mathrm{~mm}$, it stretches from $\operatorname{Re}=2.16 \times 10^{5}$ to $\operatorname{Re}=3.04 \times 10^{5}$. Nevertheless, the maximum lift values caused by the different cross-sectional shape deformations at this cable orientation are quite similar.

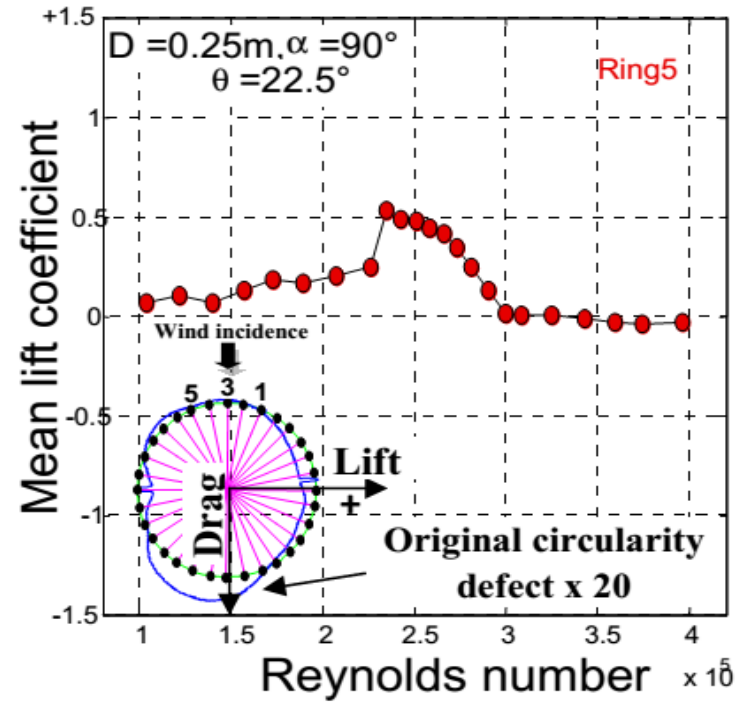

(a) $\theta=22.5^{\circ}$

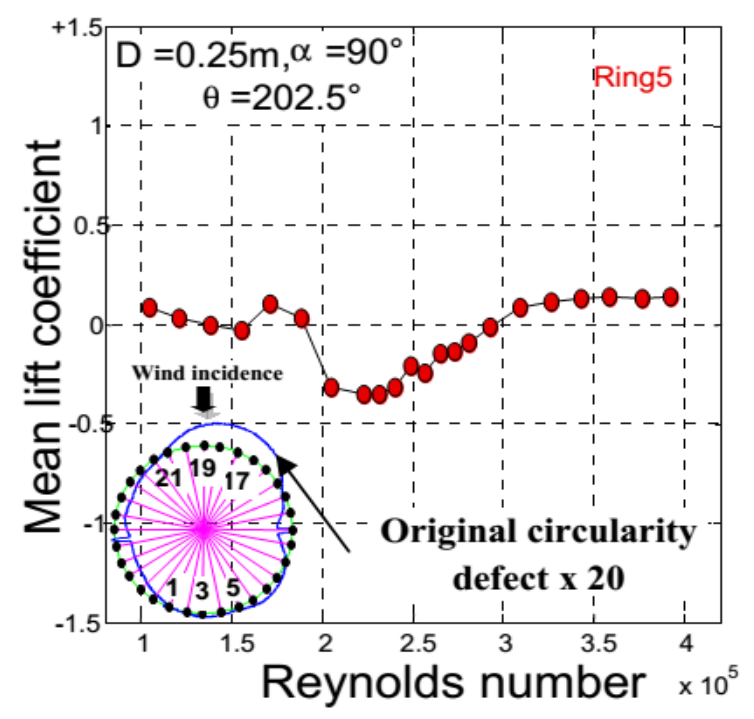

(b) $\theta=202.5^{\circ}$

Figure 4: Mean lift coefficient vs Reynolds number, original circularity defect, ring 5.

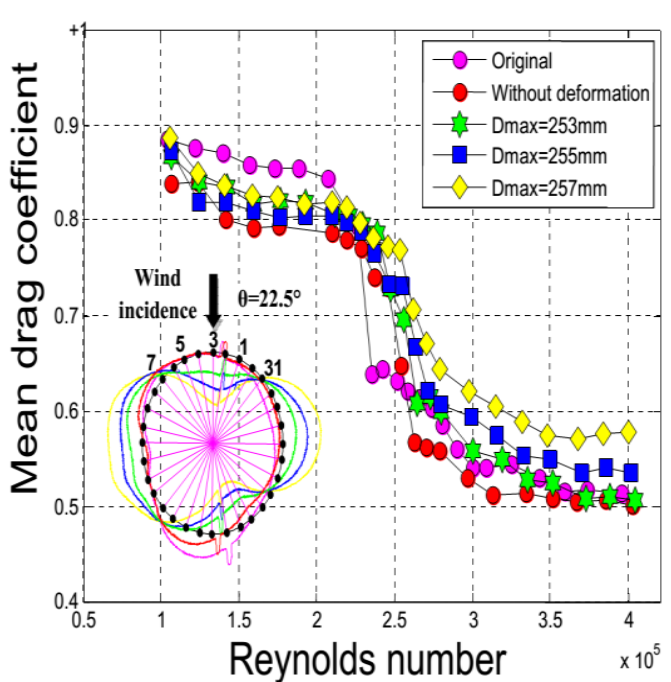

(a) Drag coefficient

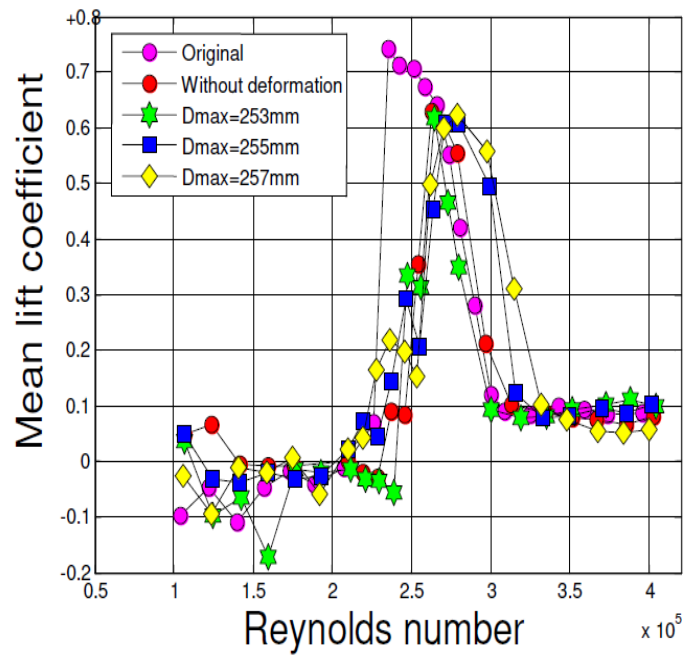

(b) Lift coefficient

Figure 5: Mean drag and lift coefficient vs. Reynolds number. $\theta=22.5^{\circ}$, Ring 3. 
The results presented in this section show that both the original circularity defect and imposed shape distortion of the sheath with helical fillet can adversely impact its aerodynamic behavior. The Reynolds number range of the critical regime becomes wider, which means that non-zero mean lift is generated at a wider range of wind velocities. In addition, for a specific cable orientation, the mean drag coefficient increases as the circularity defect grows.

\subsubsection{Smooth surface}

The impact of natural circularity defect on smooth cable aerodynamics has been previously reported by Benidir et al. (2015). This section discusses the impact of artificially increasing the macroscopic defect on cable aerodynamics. Figure $\underline{6}$ shows the variation of the mean lift coefficient as a function of Reynolds number. For the initial (without deformation) circularity defect, the first transition from the TrBL0 to the TrBL1 regime measured by most of the pressure rings along the cable occurs on the same side of the sheath, as illustrated in figure $\underline{6}-a$. However, the maximum of the lift force acting on each ring differs from one location to another. For instance, the discrepancy between the maximum lift coefficient at rings number 2 and 3 is around $50 \%$. Furthermore, the establishment of the single bubble regime occurs at the same wind velocity, which suggests the presence of a time and spatial correlation between the pressures captured around the rings. It should also be noted that the maximum lift occurs at $\operatorname{Re}=3 \times 10^{5}$ for the undeformed sheath; this Reynolds number decreases gradually to $2.5 \times 10^{5}$ as the imposed deformation increases.

The same conclusion can be drawn for the sheath with Dmax $=253 \mathrm{~mm}$ (graph $\underline{6}-\mathrm{b}$ ), for which the transition occurs on the same side of the model. This result can be justified by the fact that the first step of imposed circularity defect was in the same order of magnitude as the initial circularity defect. Nevertheless, the lift forces generated from the rings at the critical regime are lower than in the case of the initial shape. For the second and third steps of shape distortion, a sign change occurs on the mean lift coefficient. An important area of the cable surface is concerned, as illustrated in figure $\underline{6}-\mathrm{c}$ and $\underline{6}-\mathrm{d}$. For Dmax $=255 \mathrm{~mm}$, the pressure pattern of five rings has been modified, which means that the transition from the TrBL0 to the TrBL1 regime takes place on the other side of the model compared to the case of the initial shape or Dmax $=253 \mathrm{~mm}$. This change was confirmed for the largest deformation, where the same area of the model is prone to the same inversion of the local lift force. It is important to note that the wind blows towards tap 3 for all tests, i.e. $\theta=22.5^{\circ}$. As a conclusion, cable shape distortion can considerably affect the pressure pattern around the smooth circular cylinder especially in the critical regime, resulting in major changes in the mean lift coefficient. 
Smooth surface

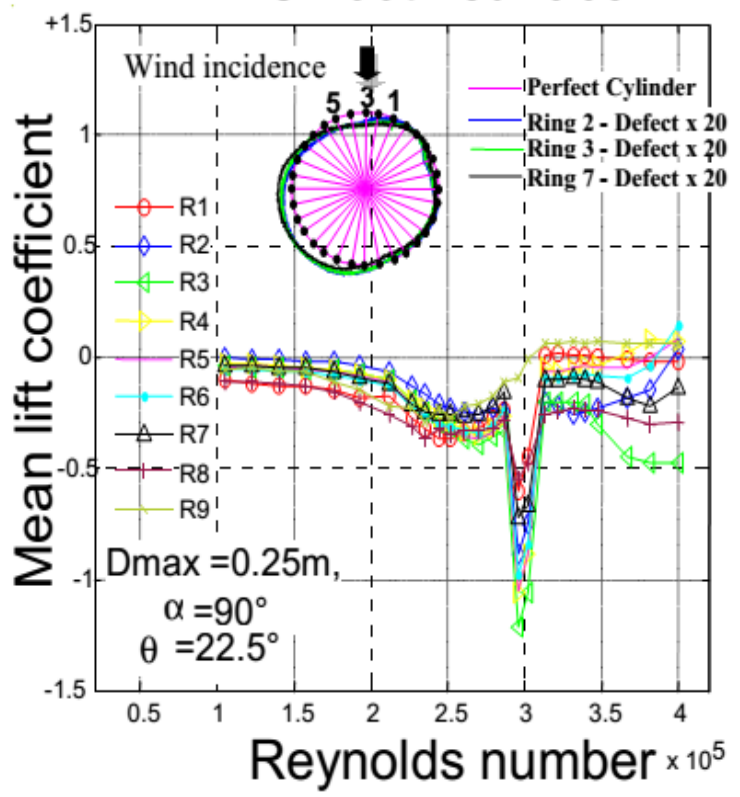

(a) Initial circularity defect

Smooth surface

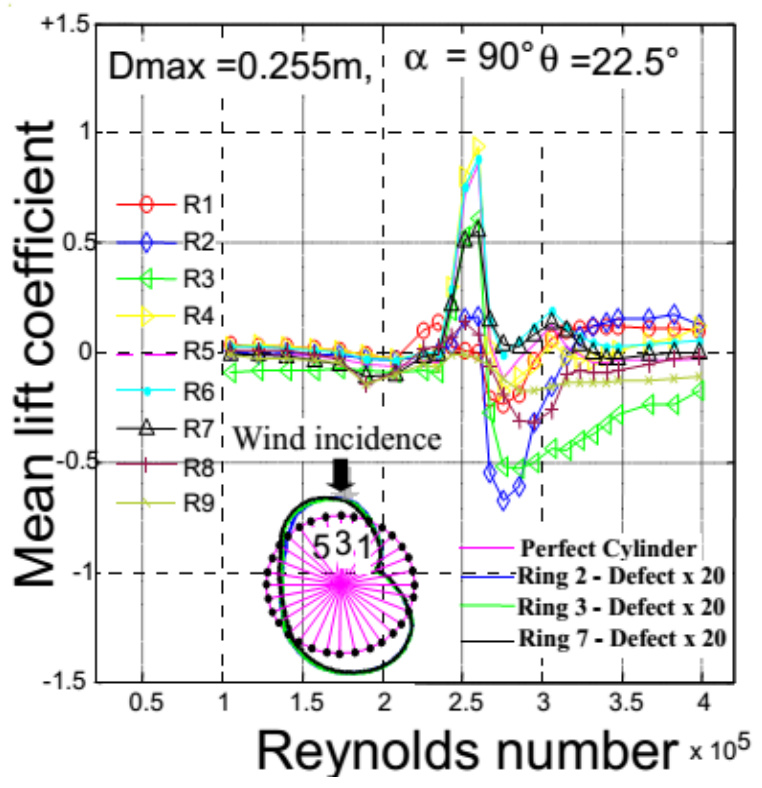

\section{Smooth surface}

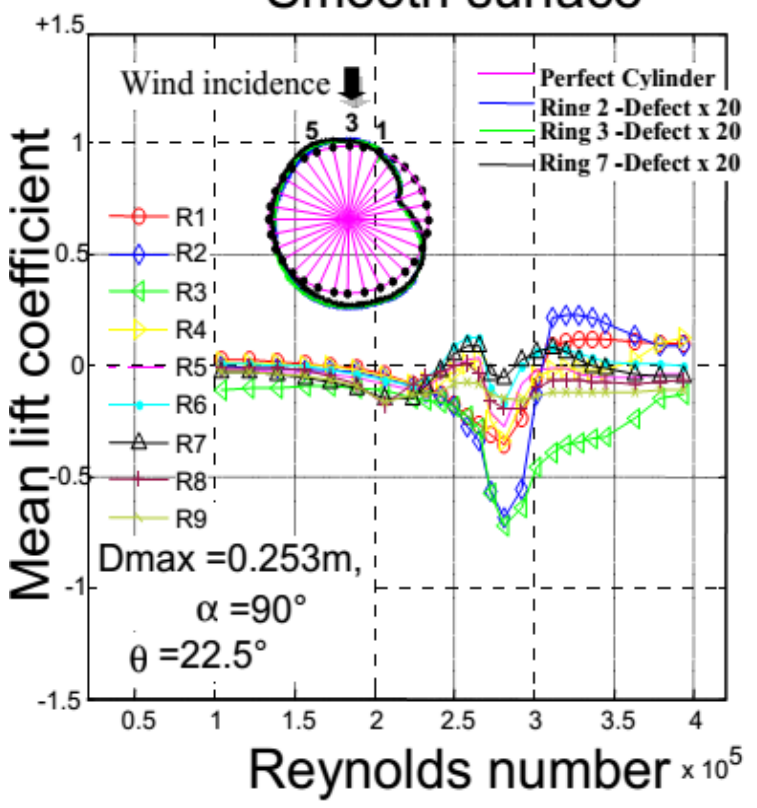

(b)Dmax $=253 \mathrm{~mm}$

Smooth surface

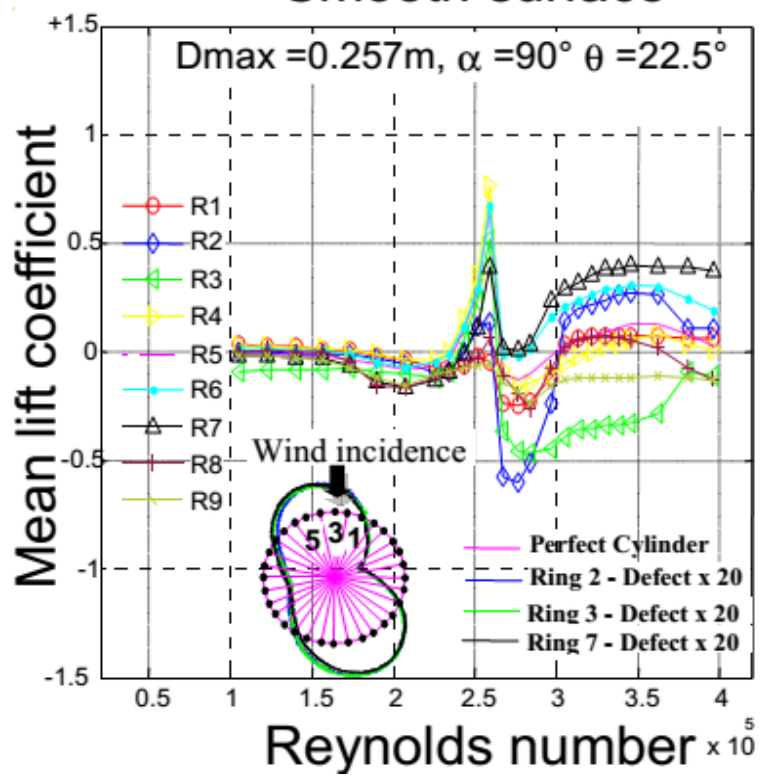

(c) $\operatorname{Dmax}=255$

(d) Dmax $=257 \mathrm{~mm}$

Figure 6 : Mean lift coefficient vs. Reynolds number, $\theta=22.5^{\circ}$.

\subsection{Bi-stability}

In the transitional Reynolds number range, the flow around the sheaths was often bi-stable; over a long time duration the flow could jump between different boundary layer transition regimes, resulting in abrupt jumps in the value of the lift. The mean lift coefficient plots on figures $\underline{4}-\underline{6}$ do not contain any information on bi-stability, as the plotted data are time averages. Conversely, figures $\underline{7}-10$ plot the 
instantaneous lift coefficient acting on the rings of the sheath with helical fillet, for increasing imposed deformation. The Reynolds numbers corresponding to each figure are respectively $R e=2.35 \times 10^{5}$, $2.42 \times 10^{5}, 2.51 \times 10^{5}$ and $2.51 \times 10^{5}$ for the non-deformed sheath, for Dmax $=253 \mathrm{~mm}$, for Dmax $=$ $255 \mathrm{~mm}$ and for Dmax $=257 \mathrm{~mm}$. In all cases the wind incidence is $\theta=22.5^{\circ}$. The duration of each signal is $60 \mathrm{~s}$; during this time, the lift on several of the rings jumps more than once between two values. One of the values is close to zero, i.e. the corresponding flow is nearly symmetric, while the other value is either positive or negative, i.e. the corresponding flow is asymmetric. The jumps are not always synchronized on all the rings and there are some rings where no jumps occur. Consequently, in this Reynolds number range, the flow can locally switch between different transition regimes (TrBL0 to TrBL1).

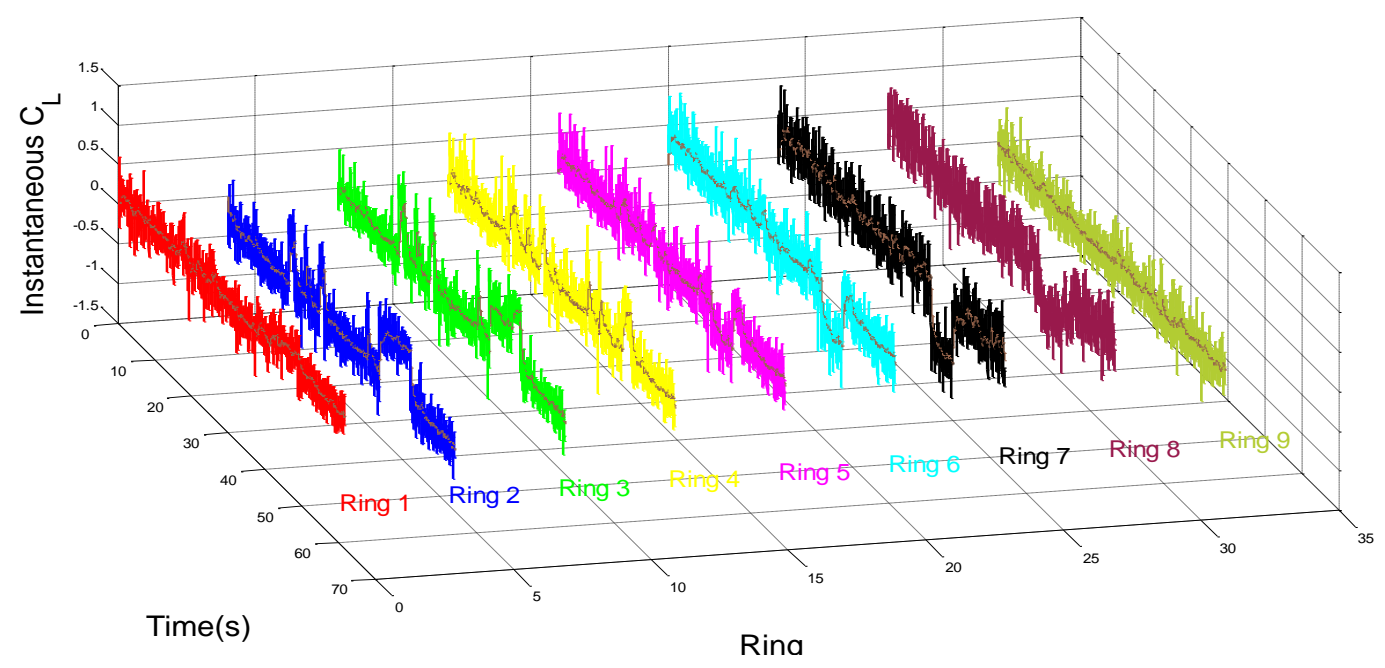

Figure 7: Instantaneous lift coefficient, helical fillet cable sheath, $\mathrm{Re}=2.35 \times 10^{5}, \theta=22.5^{\circ}$.

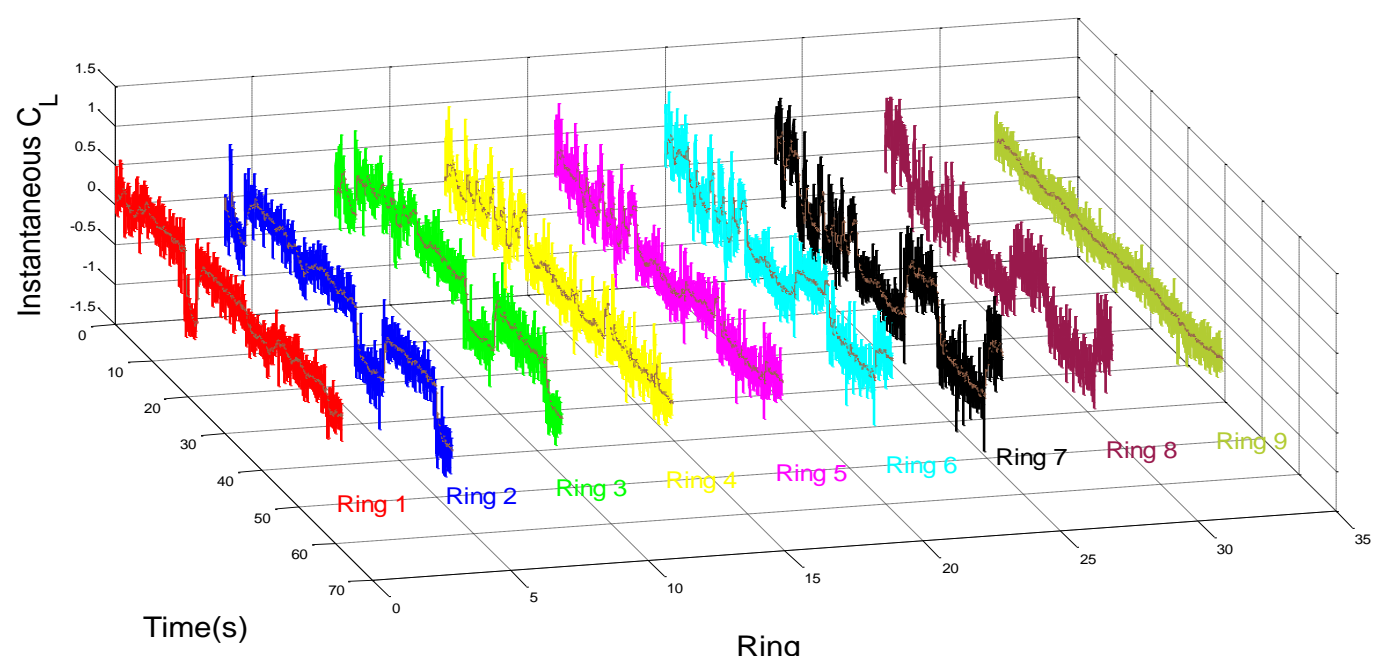

Figure 8: Instantaneous lift coefficient, helical fillet cable sheath, Dmax $=253 \mathrm{~mm}, \operatorname{Re}=2.42 \times 10^{5}$, $\theta=22.5^{\circ}$. 


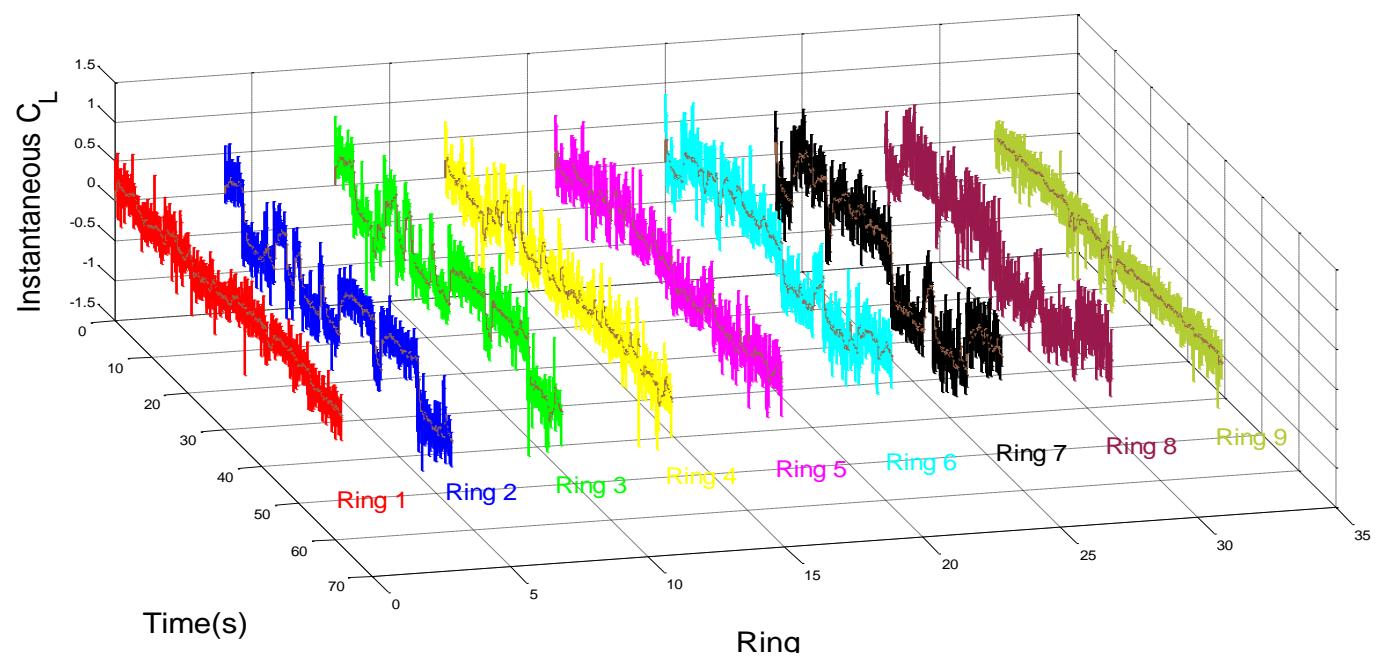

Figure 9: Instantaneous lift coefficient, helical fillet cable sheath, Dmax $=255 \mathrm{~mm}, \operatorname{Re}=2.51 \times 10^{5}$, $\theta=22.5^{\circ}$.

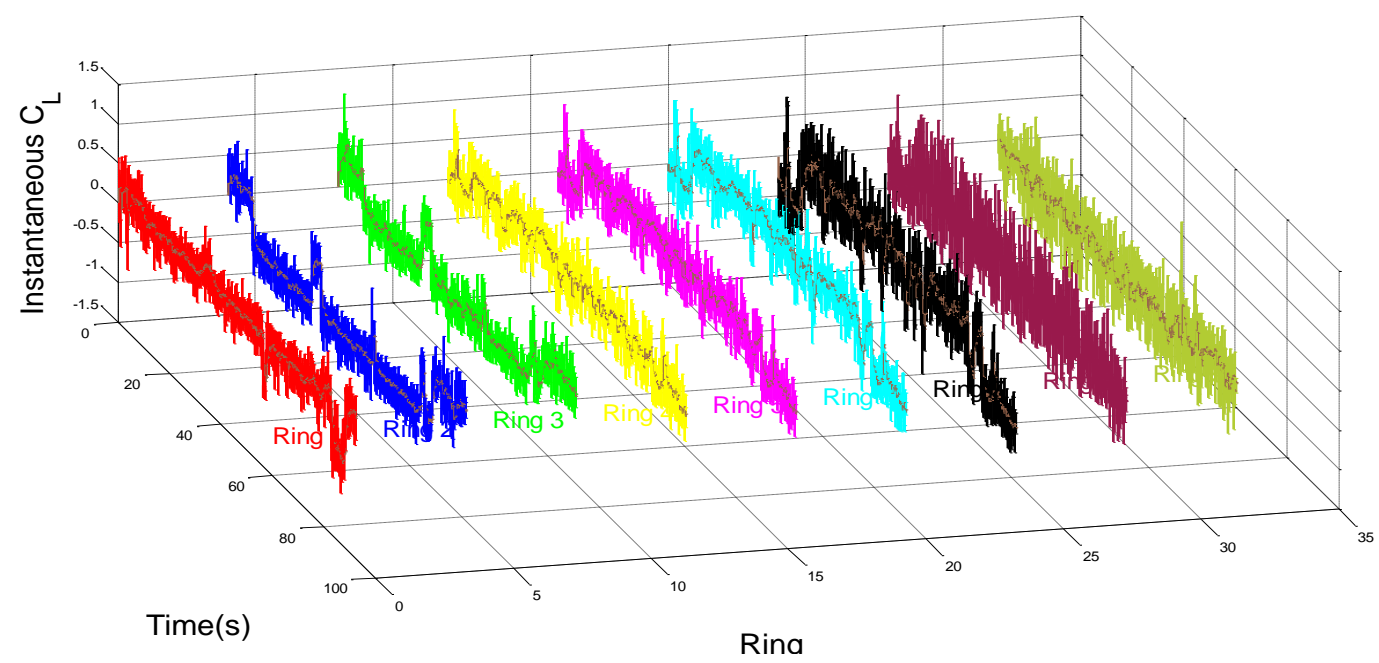

Figure10: Instantaneous lift coefficient, helical fillet cable sheath, Dmax $=257 \mathrm{~mm}, \operatorname{Re}=2.51 \times 10^{5}$, $\theta=22.5^{\circ}$.

The occurrence of bi-stability was analyzed using a clustering algorithm in order to identify the total number of different quasi-stable states of the lift coefficient on each ring and at each Reynolds number (see Benidir et al. (2015)). Based on the k-means technique, the clustering algorithm partitions an $\mathrm{N}$ dimensional set of data points into $\mathrm{k}$ sets, called clusters. The natural partition of the data sets (cluster numbers) corresponds to the minimization of the Davies-Bouldin (1979) parameter as defined by

$$
D B=\frac{1}{k} \sum_{i=1}^{k} \max _{j \neq i}\left\{D_{i, j}\right\}
$$

where $D_{i, j}$ is the within-to-between clusters distance for the $i^{\text {th }}$ and $\mathrm{j}^{\text {th }}$ clusters, determined from : 


$$
D_{i, j}=\frac{\bar{d}_{\imath}+\bar{d}_{J}}{d_{i, j}}
$$

where $\bar{d}_{i}$ and $\bar{d}_{j}$ are respectively the average distance between each point and the centroid of the $\mathrm{i}^{\text {th }}$ and $\mathrm{j}^{\text {th }}$ centroid of clusters and $\mathrm{d}_{\mathrm{i}, \mathrm{j}}$ is the Euclidean distance between the centroids of the $\mathrm{i}^{\text {th }}$ and $\mathrm{j}^{\text {th }}$ clusters. More details about the k-means algorithm and cluster separation criterion can be found in MacQueen (1967). Plotting all the different identified clusters at each tested Reynolds number results in a bifurcation diagram.

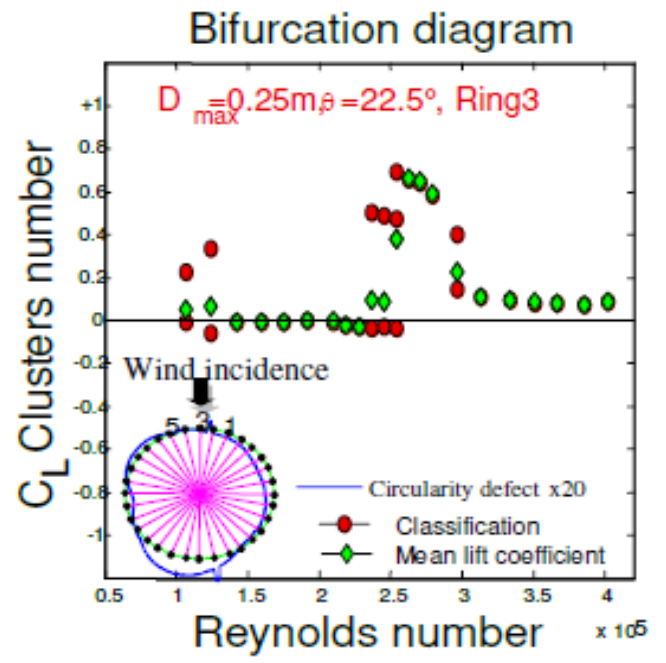

(a) Initial circularity defect

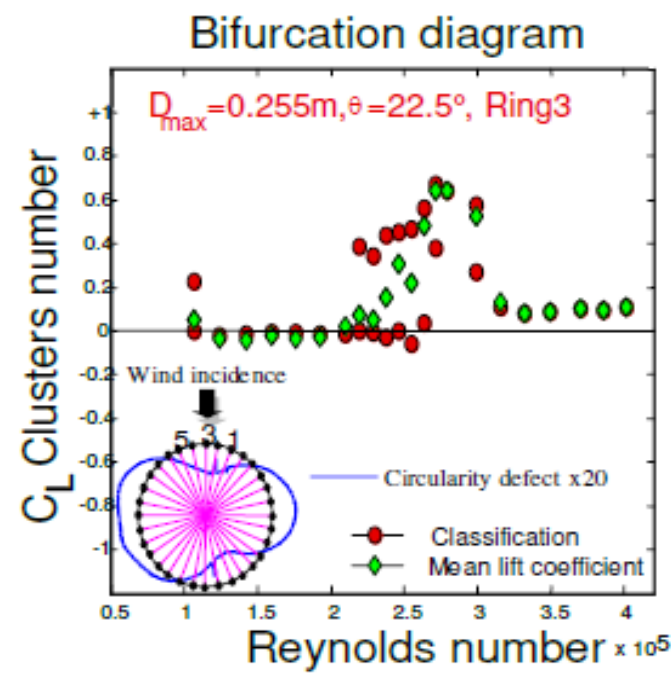

(c) $\operatorname{Dmax}=255 \mathrm{~mm}$

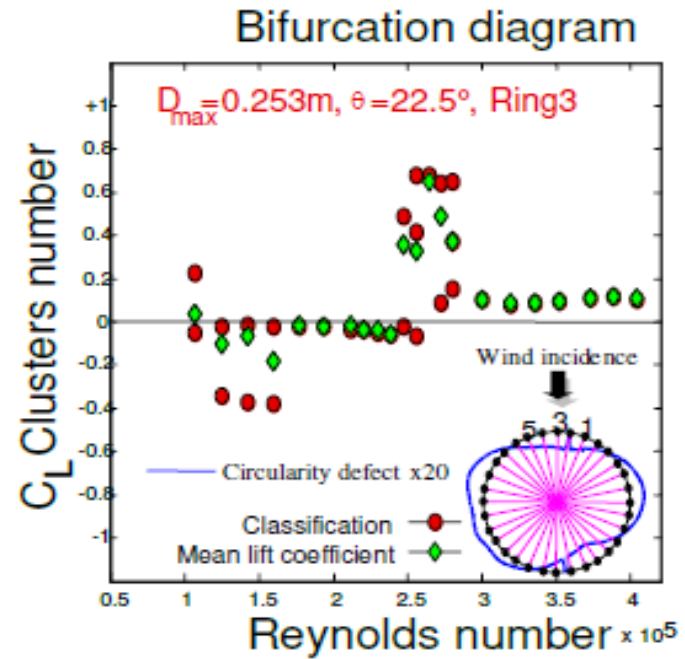

(b) $\operatorname{Dmax}=253 \mathrm{~mm}$

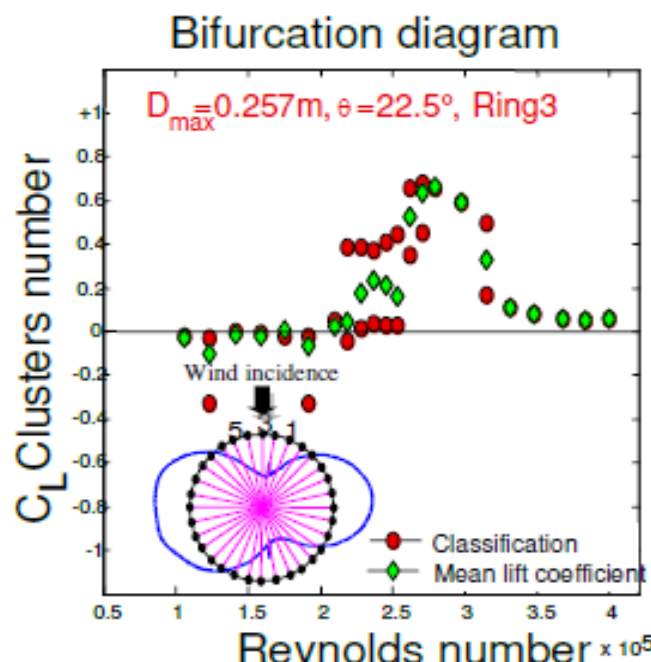

(d) $\operatorname{Dmax}=257 \mathrm{~mm}$

Figure 11: Bifurcation diagram for helical fillet cable surface, Ring 3.

Figures 11 -a to 11 -d plot the bifurcation diagrams of the instantaneous lift coefficient around ring 3 for the sheath with a helical fillet at wind incidence $\theta=22.5^{\circ}$ and for four different imposed deformations. Bi-stability occurs in a wider Reynolds number range as the circularity defect increases. For the sheath with natural circularity defect and with $\operatorname{Dmax}=253 \mathrm{~mm}$, bi-stability occurs in the $\operatorname{Re}=2.5 \times 10^{5}-3 \mathrm{x}$ 
$10^{5}$ range (figures $\underline{7}$-a and $\underline{7}-\mathrm{b}$ ). For the sheaths with $\mathrm{Dmax}=255 \mathrm{~mm}$ and $\mathrm{D}=257 \mathrm{~mm}$, the bi-stable region extends from $\operatorname{Re}=2.0 \times 10^{5}$ to $3.1 \times 10^{5}$ (figure 7 -c and $\underline{7}$-d).

The effect of both wind incidence and Reynolds number on the occurrence of bi-stability was investigated through the definition of a bi-stability percentage, defined as the number of Re values at which bi-stability occurred for each ring and each wind incidence, divided by the total number of $\operatorname{Re}$ values tested. The Reynolds number ranges from $1.7 \times 10^{5}$ to $3 \times 10^{5}$, including both the TrBL0TrBL1 and TrBL1-TrBL2 transitions. Thirteen different Re values were tested in this range. As an example, figure 11-a shows that bi-stability was encountered at four different Re values in the range $1.7 \times 10^{5}-3 \times 10^{5}$ for ring 3 of the undeformed sheath, hence the bi-stability percentage is $30.7 \%$ at this ring for $\theta=22.5^{\circ}$. Figure $\underline{11}$-c shows that, for $\operatorname{Dmax}=255 \mathrm{~mm}$, the bi-stability percentage increases to $61.5 \%$ at the same conditions.

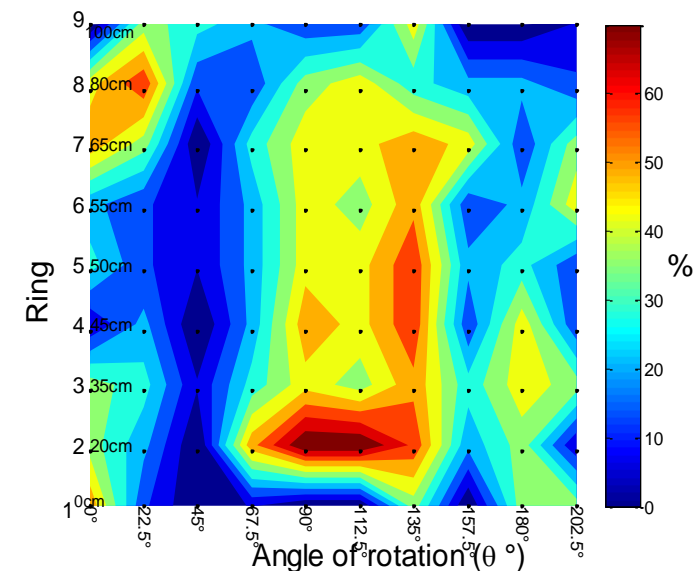

(a) Initial circularity defect

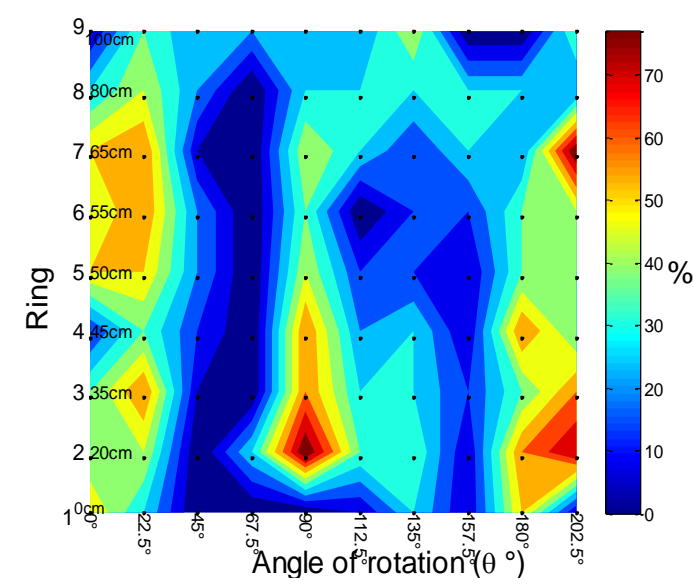

(c) $\operatorname{Dmax}=255 \mathrm{~mm}$

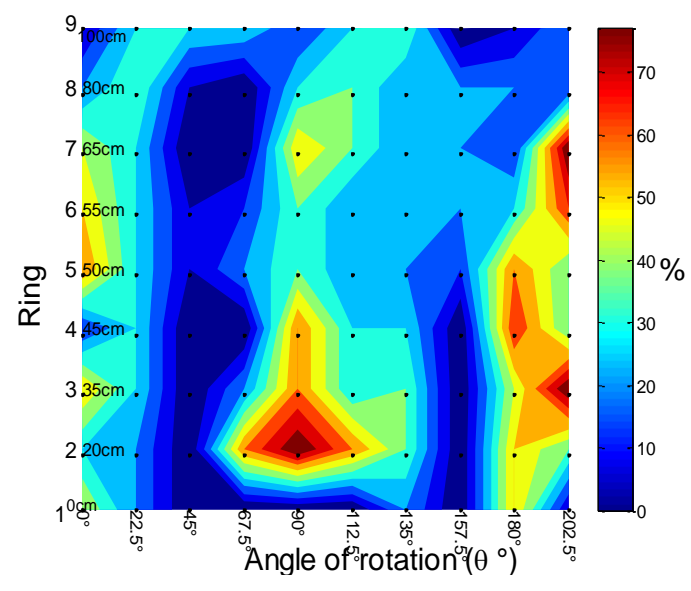

(b) Dmax $=253 \mathrm{~mm}$

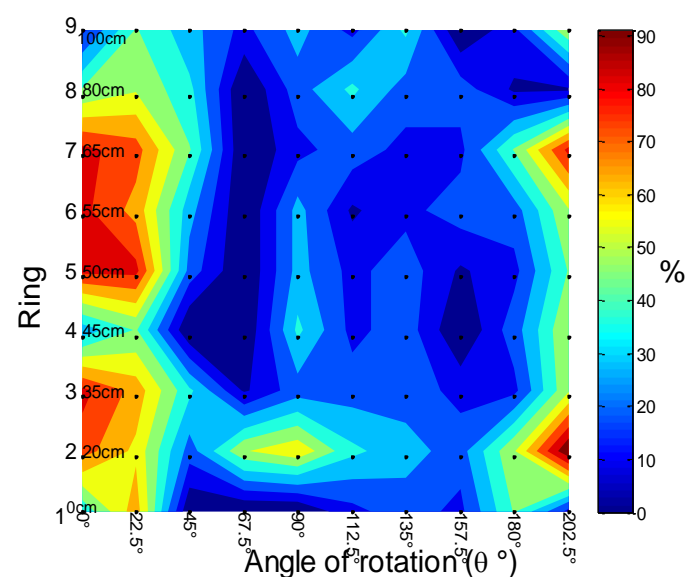

(d) $\operatorname{Dmax}=257 \mathrm{~mm}$

Figure 12: Evolution of the bi-stability when increasing the circularity defect for helical fillet cable sheath, $\mathrm{D}=250 \mathrm{~mm}, \theta$ from $0^{\circ}$ to $202.5^{\circ}$.

Figure 12 presents contour plots of the bi-stability percentage, plotted against wind incidence (horizontal axis) and ring number (vertical axis). The wind incidence ranged from $0^{\circ}$ to $202.5^{\circ}$. The four subplots concern different imposed deformations for the sheath with a helical fillet. For the undeformed sheath (figure 12-a) the highest bi-stability percentages occur at wind incidences between 
$\theta=90^{\circ}$ and $135^{\circ}$ and in rings $2-7$. However, as the deformation is increased and the cross-sectional shape is distorted, the bi-stability percentage at these wind incidences is seriously reduced. The bistability is gradually shifted towards incidences in the ranges $0^{\circ}-22.5^{\circ}$ and $180^{\circ}-202.5^{\circ}$. Furthermore, the maximum bi-stability percentage is increased up to $90 \%$, which means that bi-stable phenomena can be observed in nearly the entire Reynolds number range between $1.7 \times 10^{5}$ and $3 \times 10^{5}$ for some of the rings. The rings most affected by bi-stability are rings 2-7 for all values of the imposed deformation; only the undeformed sheath undergoes bi-stability at ring 8 . Furthermore, the length of the sheath affected significantly by bi-stability increases with the imposed deformation. For Dmax = $257 \mathrm{~mm}$ and $\theta=0^{\circ}$ this length exceeds $30 \mathrm{~cm}$ in total.

\subsection{Pressure distribution around the sheath with helical fillet}

The unsteady pressure distribution around the sheath with helical fillet is analyzed using Proper Orthogonal Decomposition (POD). This approach was already applied to a smooth sheath in Benidir et al.(2015); more details about it can be found in Andrianne et al. (2011) and Dowell et al. (1998). POD is a procedure for extracting an orthogonal basis for modal decomposition from an ensemble of signals. It relies on statistical processing of the signals to extract some coherence or organization. It is a very powerful tool in the sense that the projection on the subspace used to model the signals will contain the most energetic modes.

The data recorded from the pressure taps along the tube can be arranged into a matrix $P(N, M)$, where $\mathrm{N}$ and $\mathrm{M}$ represent respectively the number of pressure taps and the number of time instances,

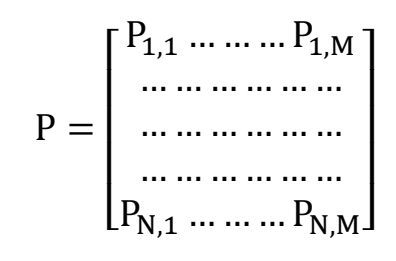

The pressure $P(x, t)$ can be written as

$$
P(x, t)=\sum_{i=1}^{N} \varphi_{i}(x) q_{i}(t)
$$

$\varphi_{i}(x)$ are the spatial POD mode shapes and $q_{i}(t)$ are the generalized coordinates, which are functions of time. The POD procedure makes use of the eigenvalue decomposition

$$
C A=\lambda A
$$

where $C$ is the covariance matrix of $P$, while $A$ and $\lambda$ are respectively the matrices of eigenvectors and eigenvalues of $C$. The energy of the POD modes was ranked using the Cumulative Percentage Variance (CPV) criterion. The formulation of this criterion is given by

$$
C P V(I)=100 \frac{\sum_{j=1}^{I} \lambda_{j}}{\sum_{j=1}^{N} \lambda_{j}} \%
$$

where $I$ is the number of retained modes. This criterion is applied to select the most energetic modes from the eigenvalues matrix. The associated generalized coordinates are calculated from

$$
q=\varphi^{T} P
$$


Figures $\underline{13}-16$ plot the results of the POD analysis for the sheath with a helical fillet at $\theta=22.5^{\circ}$ and for the different imposed deformations. The Reynolds number is specified below the figures. For each figure, subplot (a) plots $C P V(I)$, subplot (b) plots the first three generalized coordinates and subplots (c)-(e) the first three POD mode shapes. Figures 13-a, 14-a, 15-a and 16-a show that the first three modes contain more than $97 \%$ of the energy of the signals for all cases.

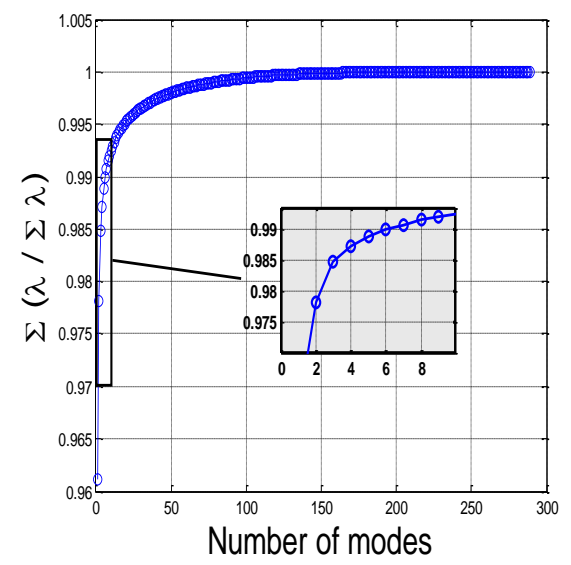

(a) Energy of the mode

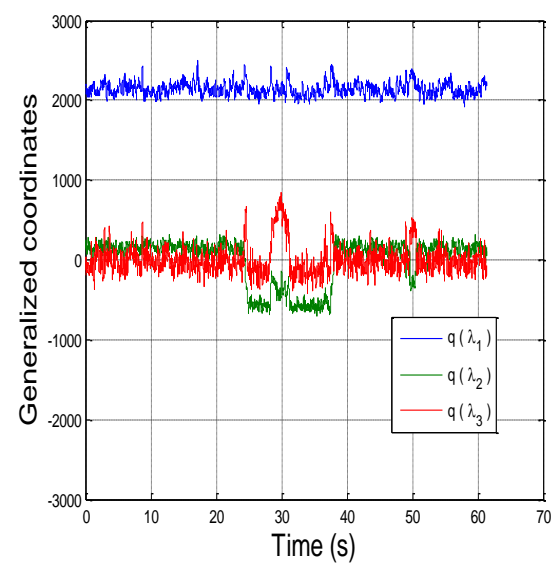

(b) Generalized coordinates

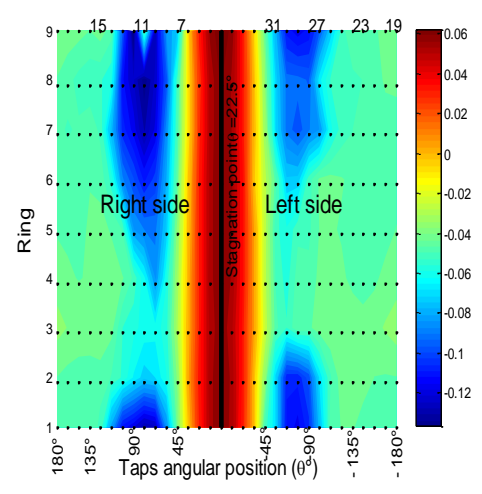

(c) Mode shape, $\lambda_{1}$

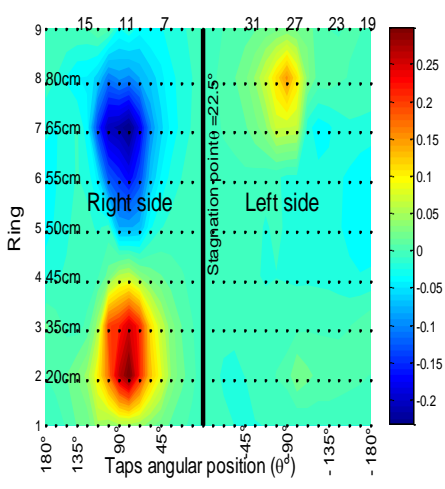

(d) Mode shape, $\lambda_{2}$

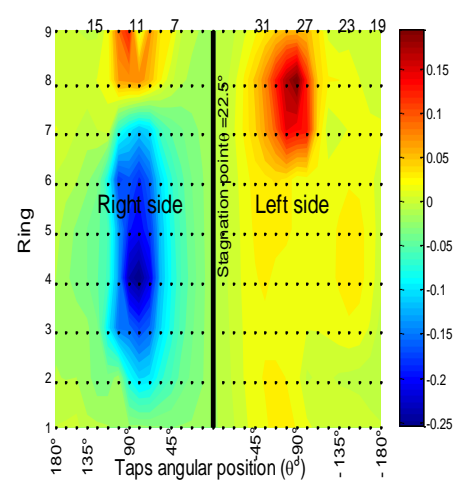

(e) Mode shape, $\lambda_{3}$

Figure 13: Proper Orthogonal Decomposition of the pressure pattern, helical fillet without deformation of the cross-sectional shape, $\operatorname{Re}=2.35 \times 10^{5}$ 


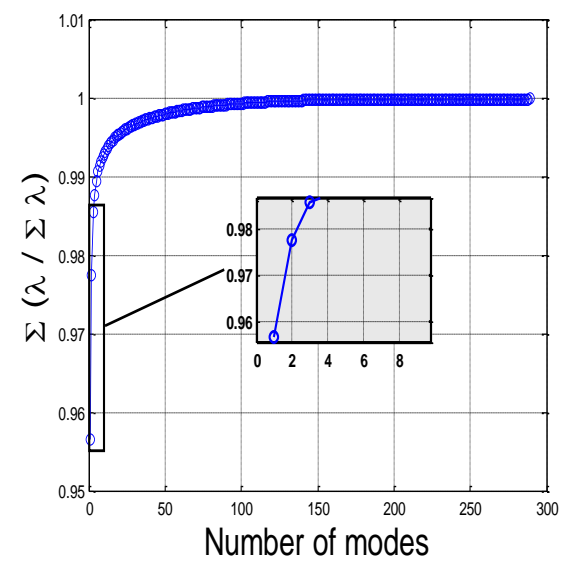

(a) Energy of the mode

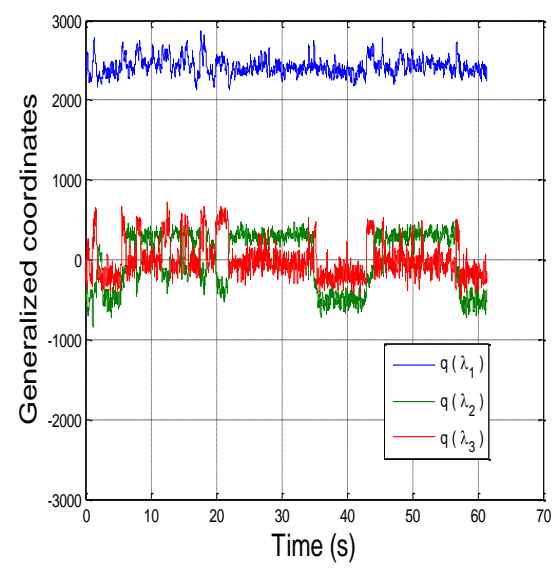

(b) Generalized coordinates

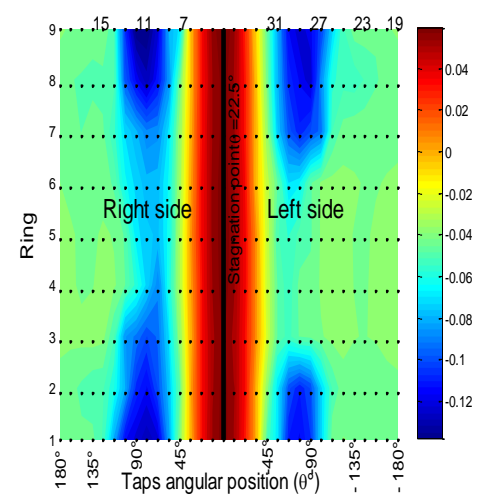

(c) Mode shape, $\lambda_{1}$

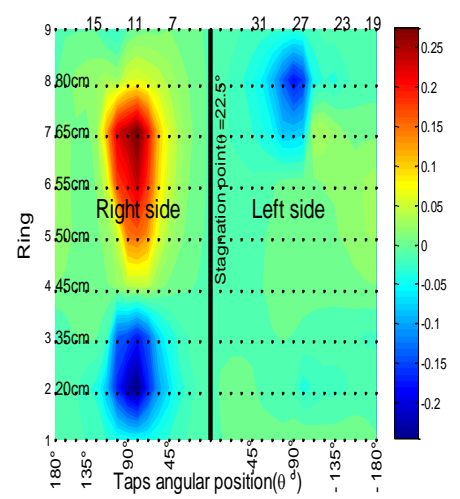

(d) Mode shape, $\lambda_{2}$

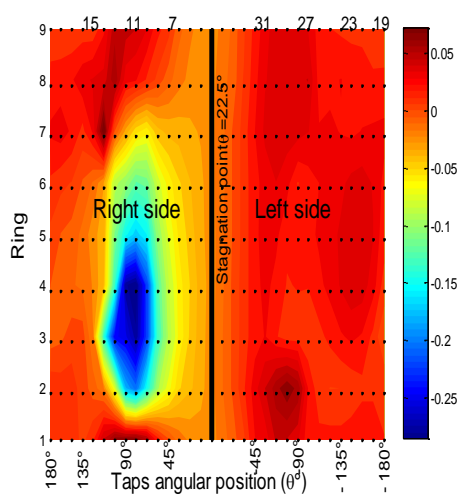

(e) Mode shape, $\lambda_{3}$

Figure 14 : Proper Orthogonal Decomposition of the pressure pattern, helical fillet Dmax $=253 \mathrm{~mm}$,

$$
\operatorname{Re}=2.42 \times 10^{5}
$$

Mode 1 in all cases represents the mean flow, as the generalized coordinate corresponding to this mode is constant at all times. It can be seen that this flow is nearly but not completely symmetric for all imposed deformations and even for the undeformed sheath. This asymmetry of the mean flow is due to the presence of the helical fillet and was observed for all wind incidences and Reynolds numbers.

Asymmetry due to turbulent transition in the boundary layers is represented by modes 2 and 3. At time instances when the corresponding generalized coordinates are equal to zero, the flow is equal to the mean flow and there is steady asymmetry due to the helical fillet. At times when the generalized coordinates of modes 2 and 3 are non-zero, the flow undergoes transition, becomes temporarily asymmetric and generates lift. Mode 2 always features an area of high pressure and an area of low pressure on the right side, along with a small area of high or low pressure around ring 8 on the left side. Mode 3 features a single area of high/low pressure on the right side, except for Dmax $=257 \mathrm{~mm}$ where there are two distinct areas of high pressure on the right. 


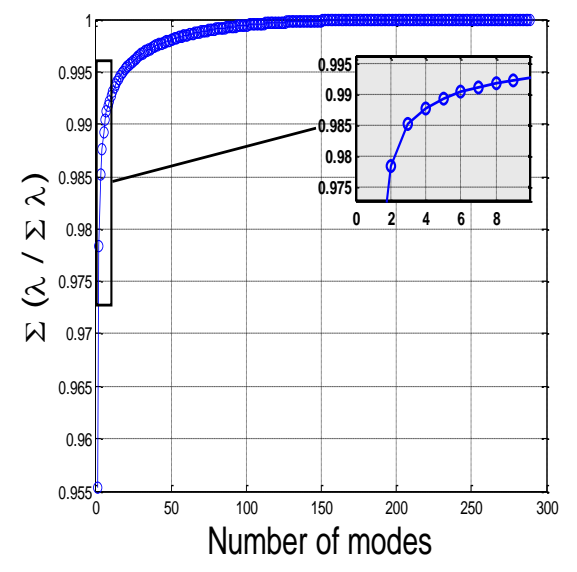

(a) Energy of the mode

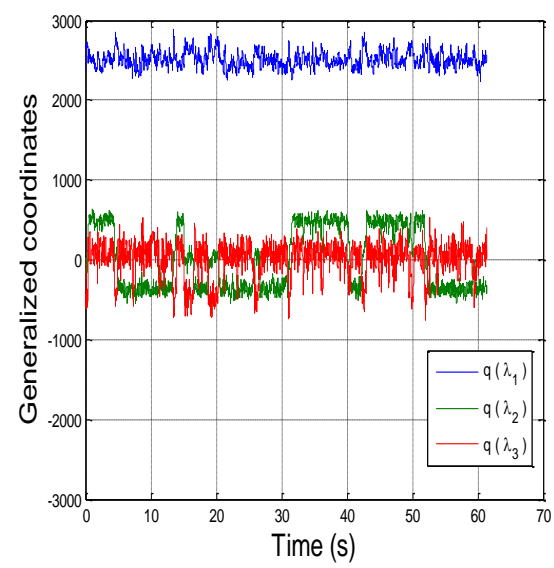

(b) Generalized coordinates

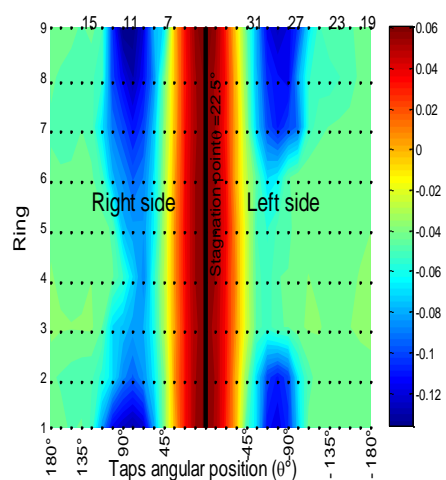

(c) Mode shape, $\lambda_{1}$

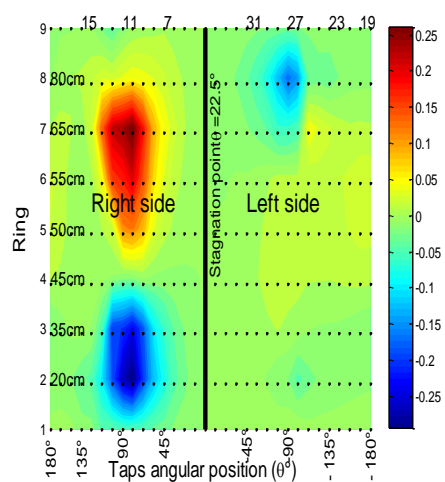

(d) Mode shape, $\lambda_{2}$

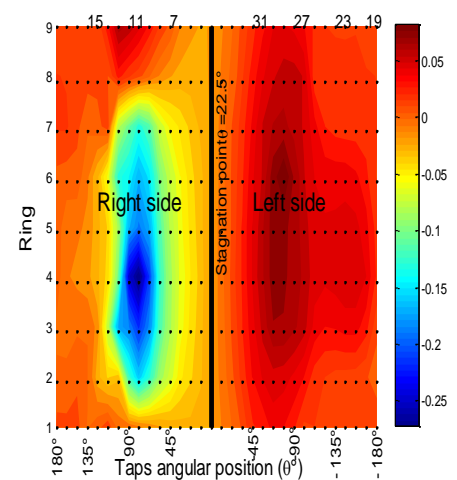

(e) Mode shape, $\lambda_{3}$

Figure 15 : Proper orthogonal decomposition of the pressure pattern, helical fillet Dmax $=255 \mathrm{~mm}$,

$$
\operatorname{Re}=2.51 \times 10^{5}
$$

Increasing the imposed deformation has a significant effect on the response of the POD modes. For the undeformed case, the generalized coordinates of modes 2 and 3 are equal to zero most of the time but jump to non-zero values over a 10 -second period in the middle of the time record (see figure $13-\mathrm{b}$ ). In contrast, figures $\underline{14}-\mathrm{b}, \underline{15}-\mathrm{b}$ and $\underline{16}-\mathrm{b}$ show that the generalized coordinate for mode 2 is nearly always non-zero but jumps between positive and negative values. This means that the regions of high or low pressure lying on the right side of the sheath change sign regularly throughout the time record. During some time periods rings 2-3 see low pressure while rings 5-8 see high pressure on the right side; during other time periods rings 2-3 see high pressure and rings 5-8 low pressure. The generalized coordinate of mode 3 is still mostly equal to zero but jumps to non-zero values more often when deformation is imposed. When this happens, rings 2-8 feature high or low-pressure bubbles on the right side. This means that up to $60 \mathrm{~cm}$ of the length of the sheath can be affected by bi-stable phenomena when deformation is imposed.

Every time the generalized coordinates of modes 2 and 3 jump in value, the lift changes magnitude and/or direction. Such changes constitute significant impulses applied on the sheath that will cause structural displacement in flexible cables. Sometimes, the changes in the flowfield are synchronized over the entire instrumented length of the sheath, causing a uniform lift to the left or to the right. Consider the case Dmax $=255 \mathrm{~mm}$ plotted in figure 15 . At times when the generalized coordinates of both modes 2 and 3 are positive, there is a uniform high-pressure area over most of the right side of 
the sheath (rings 3-5), which cancels the low pressure caused by mode 1. A net lift towards the left will be applied on the majority of the sheath, causing a significant impulse when the jump occurs. Nevertheless, figures $\underline{13}-\underline{16}$ show that, for the sheath with a helical fillet, synchronized changes in the flow field that cause a net lift towards the same direction over the entire length of the sheath are rare. In most time instances, the generalized coordinates of modes 2 and 3 have incompatible signs or one of them is zero. In such cases, the lift pushes half of the sheath towards the left and the other half towards the right (or vice-versa). This means that, when jumps occur, the resulting impulse acting on the sheath is weaker and the associated structural response is expected to be less pronounced.

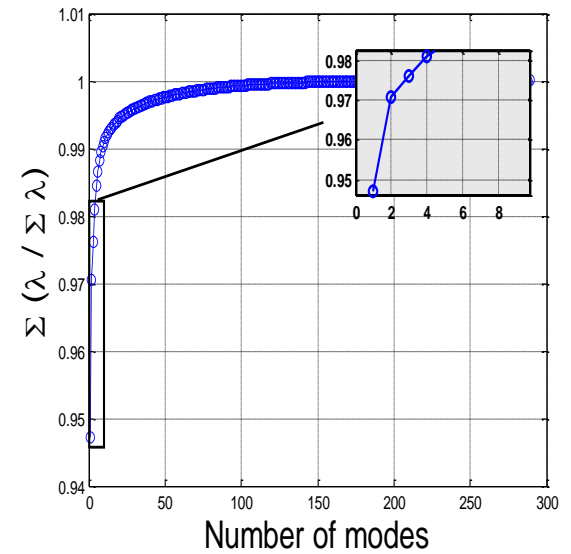

(a) Energy of the mode

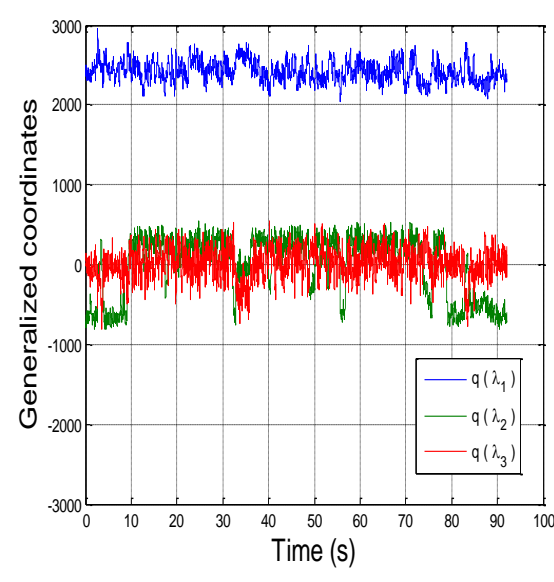

(b) Generalized coordinates

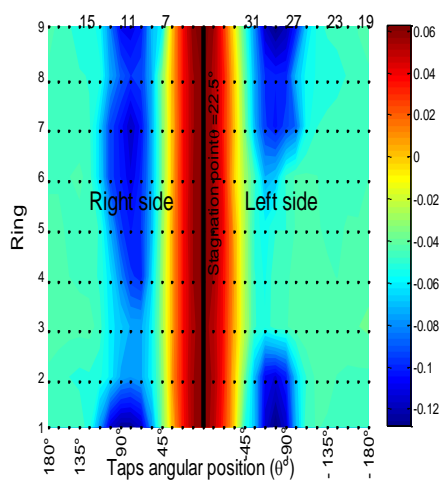

(c) Mode shape, $\lambda_{1}$

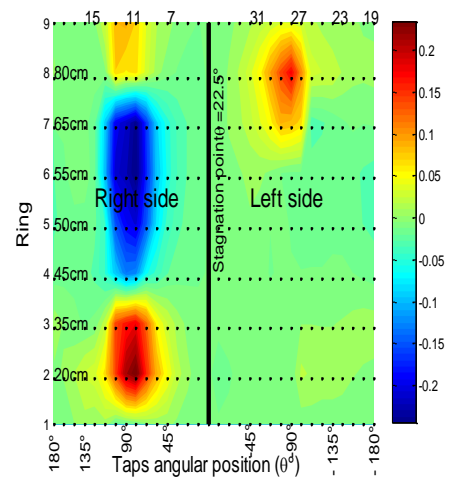

(d) Mode shape, $\lambda_{2}$

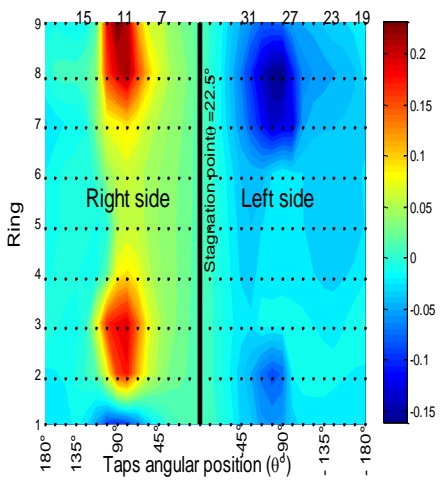

(e) Mode shape, $\lambda_{3}$

Figure 16: Proper orthogonal decomposition of the pressure pattern, helical fillet Dmax $=257 \mathrm{~mm}$,

$$
\operatorname{Re}=2.51 \times 10^{5}
$$

For the case $\mathrm{D}=257 \mathrm{~mm}$ in particular (figure 16 ), the generalized coordinate of mode 3 is zero almost exclusively when that of mode 2 is not equal to zero. Therefore, there are no time instances when modes 2 and 3 cause a uniform low or high-pressure bubble over the entire length of the sheath. The energy of mode 1 is lower than in the other deformation cases and that of mode 2 is higher, which means that bi-stability is more important. Yet, jumps in the values of the generalized coordinates are less frequent and result in non-uniform changes in pressure along the length of the sheath. Hence, the effect of bi-stability is expected to be less pronounced on the structural response of the cable. 


\section{CONCLUSION}

This paper reports on the effect of increasing the circularity defect on the sheaths of cables for cablestayed bridges. For smooth sheaths, imposing deformation on their cross-section shifts the critical regime to lower Reynolds numbers. For sheaths with helical fillets, the critical Reynolds number range becomes wider. Important bi-stable phenomena are observed for both types of sheath in the critical regime, causing significant jumps in instantaneous lift.

Imposed deformation has a mixed effect on the aerodynamic stability of the flow around the sheath with helical fillet. On the one hand it increases the Reynolds number range of the critical regime and makes bi-stable phenomena more pronounced. On the other hand, the helical fillet de-correlates the flow, resulting in regions of opposite pressure on the same side of the sheath, even at the highest imposed deformation. This means that jumps in lift are rarely uniform over the entire length of the sheath and any resulting impulses will not have a coherent effect on the structural response of the enclosed cable. This result could be taken to mean that the introduction of a helical fillet has a positive effect on dry galloping stability, as well as on rain-and-wind-induced vibration. Nevertheless, it should be kept in mind that the present experiments were carried out on sheaths of length $2 \mathrm{~m}$ and flow correlation phenomena might be different for much longer sheaths.

\section{REFERENCES}

[1] Nikitas N., Macdonald, J. H. G. 2015. Aerodynamic forcing characteristics of dry cable galloping at critical Reynolds numbers. European Journal of Mechanics B/Fluids. Vol 49, pp. 243-249.

[2] Mctavish S., Yamauchi K. and Sato H. 2017. An investigation of large-amplitude cable vibrations at critical and supercritical Reynolds numbers. International Symposium on the Dynamics and Aerodynamics of Cables (ISDAC).Oct, 30-31, FEUP, Porto, Portugal.

[3] Larose, G.L. and Zan, S.J. 2001. The aerodynamic forces on the stay cables of cable-stayed bridges in the critical Reynolds number range. Proceedings of the Fourth International Symposium on Cable Dynamics., (77-84),Montreal, Canada.

[4] Cheng, S., Irwin, P.A., Jakobsen, J. B., Lankin, J., Larose, G. L., Savage, M. G., Tanaka,H. and Zurell, C. 2003. Divergent motion of cable exposed to skewed wind. Proceedings of the 5th International Symposium on Cable Dynamics, (271-278), Santa Margherita, Italy.

[5] Kleissl, K. and Georgakis, C. T. 2012. Comparison of several innovative bridge cable surface modifications. The Seventh International Colloquium on Bluff Body Aerodynamics and Applications (BBAA7) Shanghai, China; September 2-6.

[6] Katsuchi, H. and Yamada, H. 2009. Wind-tunnel Study on Dry-galloping of Indented-surface Stay Cable. The $11^{\text {th }}$ Americas conference on wind engineering. San Juan, Puerto Rico. June 22-26.

[7] Vo H-D, Katsuchi H, Yamada H and Nishio M, 2016. A wind tunnel study on control methods for cable dry-galloping. Journal of Frontiers of Structural and Civil Engineering. Vol. 10(1), pp, 72-80. 
[8] Matsumoto M, Yagi T, Hatsuda H, Shima T, Tanaka M, Naito H. 2010. Dry galloping characteristics and its mechanism of inclined/yawed cables. Journal of Wind Engineering and Industrial Aerodynamics, 98 (6-7), pp, 317-327.

[9] Zdravkovich, M. M., 1997. Flow around circular cylinder - Volume 1: Fundamentals, pages 163198, Oxford University Press.

[10] Schewe, G., 1983. On the force fluctuations acting on a circular cylinder in cross flow from subcritical up to trans-critical Reynolds numbers. Journal of fluid Mechanics, Cambridge University Press, August-1983, 133, pp. 265-285.

[11] Nikitas, N., Macdonald, J. H. G., Jakobsen, J. B., and Andersen, T. L. 2012. Critical Reynolds number and galloping instabilities : experiments on circular cylinders. Experiments in Fluids., 52, Issue5,(1295-1306).

[12] Benidir, A., Flamand, O., Gaillet, L., Dimitriadis, G. 2014. Impact of circularity defect on helical fillets HDPE bridge stay covers: Analysis of bi-stability at critical Reynolds number by bifurcation diagrams. The $1^{\text {st }}$ Symposium on the Dynamics and Aerodynamics of Cables (SDAC) - Sept. 25-26, DTU, Copenhagen, Denmark.

[13] Flamand, O., Boujard, O., 2009. A comparison between dry cylinder and rain-wind induced excitation, Proceedings of the 5th European and African, Florence, Italy.

[14] Matteoni, G., Georgakis, C. T., 2012. Effects of bridge cable surface roughness and crosssectionaldistortion on aerodynamic force coefficients. Journal of Wind Engineering and IndustrialAerodynamics, 104-106, pp. 176-187.

[15] Benidir, A., Flamand, O., Gaillet, L., Dimitriadis, G., 2015, Impact of roughness and circularity defect on bridge cables stability. Journal of Wind Engineering and Industrial Aerodynamics, Feb. 2015, 137, pp, 1-13.

[16] Ma, W.Y., Liu, Q. K., Du, X.Q. and Wei, Y.Y., 2015. Effect of the Reynolds number on the aerodynamic forces and galloping instability of a cylinder with semi-elliptical cross sections. Journal of Wind Engineering and Industrial Aerodynamics, Volume 146, Nov. 2015, pp, 71-80.

[17] Flamand, O., 1995. Rain wind induced vibration of cables. Journal of Wind Engineering and Industrial Aerodynamics, July. 1995, Volume 57, Issue 2-3, pp, 353-362.

[18] Andrianne, T., Abdul Razak, N. and Dimitriadis, G., 2011. Flow Visualization and Proper Orthogonal Decomposition of Aeroelastic Phenomena, in Wind Tunnels, edited by Satory Okamato, ISBN 978-953-307-295-1, InTech.

[19] Dowell, E. H., Hall, K. C. and Romanowski, M. C., 1998. Eigen mode analysis in unsteady aerodynamics: Reduced order models, Applied Mechanics Reviews 50(6): 371-385.

[20] Davies, D. L., Bouldin, D. W., 1979. A cluster separation measure, IEEE transaction on patternanalysis and machine intelligence. Volume 1, Issue 2,Pages 224-227.

[21] MacQueen, J.B., 1967. Some methods for classification and analysis of multivariate observations. In: Proceedings of the Fifth Berkeley Symposium on Mathematical Statistics and Probability, 1, pp. 
281-297. University of California Press, University of California, USA, June 21-July18, 1965 and December 27, 1965-January 7, 1966, pp. 281-297. 